\title{
Supplementary Information \\ Tuning the properties of zero-field room temperature ferromagnetic skyrmions by interlayer exchange coupling
}

Roberto Lo Conte ${ }^{*}, 1,2$, Ashis K. Nandy ${ }^{*}, 3$, Gong Chen ${ }^{4}$, Andre L. Fernandes Cauduro ${ }^{5}$, Ajanta Maity $^{3}$, Colin Ophus ${ }^{5}$, Zhijie Chen ${ }^{6}$, Alpha T. N'Diaye, Kai Liu ${ }^{4,6}$, Andreas K. Schmid, ${ }^{*}$, and Roland Wiesendanger*,2

${ }^{1}$ Department of Materials Science and Engineering, University of California, Berkeley, CA 94720, USA

${ }^{2}$ Department of Physics, University of Hamburg, D-20355 Hamburg, Germany

${ }^{3}$ School of Physical Sciences, National Institute of Science Education and Research, HBNI, P.O. Jatni, 752050, India

${ }^{4}$ Department of Physics, University of California, Davis, CA 95616, USA

${ }^{5}$ National Center for Electron Microscopy, Molecular Foundry - Lawrence Berkeley National laboratory, Berkeley, CA 94720, USA

${ }^{6}$ Physics Department, Georgetown University, Washington, DC 20057, USA

${ }^{7}$ Advanced Light Source, Lawrence Berkeley National Lab, Berkeley, CA 94720, USA

*Correspondence should be addressed to: rloconte.magnetism@gmail.com;

aknandy@niser.ac.in; AKSchmid@lbl.gov; wiesendanger@physnet.uni-hamburg.de

\section{Contents:}

Section S1. First principles DFT calculations: VASP and FLEUR

Section S2. Growth of epitaxial Ir buffer layers on MgO single crystals

Section S3. Growth calibration by LEEM

Section S4. Protocol for sample preparation

Section S5. Angle distribution of the domain walls spin-texture

Section S6. Imaging of the 3D spin-texture via spin-polirized low energy electron microscopy (SPLEEM)

Section S7. Stability of IEC-stabilized ferromagnetic skyrmions against an external magnetic field

Section S8. Investigation of the interlayer exchange coupling in $\mathrm{Cu}(001) \backslash \mathrm{Ni} \backslash \mathrm{Cu}(\mathrm{t}) \backslash \mathrm{Ni} \backslash \mathrm{Fe}$

Section S9. Growth of wedged Cu spacer via shadow mask technique

Section S10. Protocol for the extraction of the skyrmions area

Section S11. Estimation of the Curie temperatures of the reference and free magnetic layers and their effect on the IEC 


\section{S1. First principles DFT calculations}

\section{VASP}

The first-principles theoretical calculations presented were carried out with density functional theory (DFT) as implemented in Vienna ab-initio Simulation Package (VASP) ${ }^{1-4}$. This is a plane wave code where electron-core interactions in pseudopotentials were described by the projector augmented wave (PAW) method $^{5,6}$ and the exchange correlation energy was calculated within the generalized gradient approximation (GGA) functional of the Perdew-Burke-Ernzerhof (PBE) form ${ }^{7}$. For structural optimizations in the film geometry, a k-point grid of $24 \times 24$ for the two-dimensional Brillouin zone (2D-BZ) and $500 \mathrm{eV}$ of plane-wave energy cut-off were employed. We investigated the magnetic properties of the materials stack $\operatorname{Ir}(10) \backslash \mathrm{Ni}(12) \backslash \mathrm{Cu}(\mathrm{x}) \backslash \mathrm{Ni}(2) \backslash \mathrm{Fe}(\mathrm{y})$, where the thicknesses of the different layers are indicated in monolayers (ML). The material system is assumed to be in a face centered cubic (fcc) configuration, with the stacking along the [001] crystallographic direction. A schematic of the simulated stack is shown in Fig. S1a where the regions mentioned by the vertical arrows are characterized by the inter-planar distances and nearest neighbor bond lengths as in Table S1b. In Fig. S1c, the regions within the vertical arrows are taken as the interface and forces are relaxed along the growth direction.

a

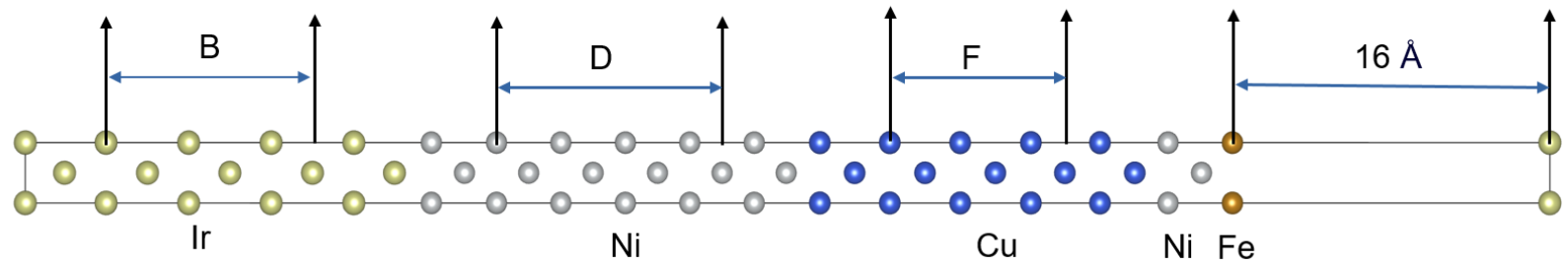

b

\begin{tabular}{|c|c|c|c|}
\hline $\begin{array}{c}\text { In plane lattice } \\
\text { constant } \\
(\boldsymbol{A})\end{array}$ & $\begin{array}{c}\text { Ir interlayer } \\
\text { distance (bond } \\
\text { length) }(\boldsymbol{A})\end{array}$ & $\begin{array}{c}\text { Ni interlayer } \\
\text { distance (bond } \\
\text { length) }(\boldsymbol{A})\end{array}$ & $\begin{array}{c}\text { Cu interlayer } \\
\text { distance (bond } \\
\text { length) }(\boldsymbol{A})\end{array}$ \\
\hline $\mathbf{2 . 7 2}\left(\mathrm{a}_{\mathrm{Ir}}\right)$ & $1.95(2.74)$ & $1.52(2.45)$ & $1.66(2.54)$ \\
\hline $\mathbf{2 . 5 5}\left(\mathrm{a}_{\mathrm{Cu}}\right)$ & $2.06(2.74)$ & $1.71(2.48)$ & $1.84(2.57)$ \\
\hline $\mathbf{2 . 4 9}\left(\mathrm{a}_{\mathrm{Ni}}\right)$ & $2.13(2.76)$ & $1.76(2.49)$ & $1.89(2.59)$ \\
\hline
\end{tabular}

C

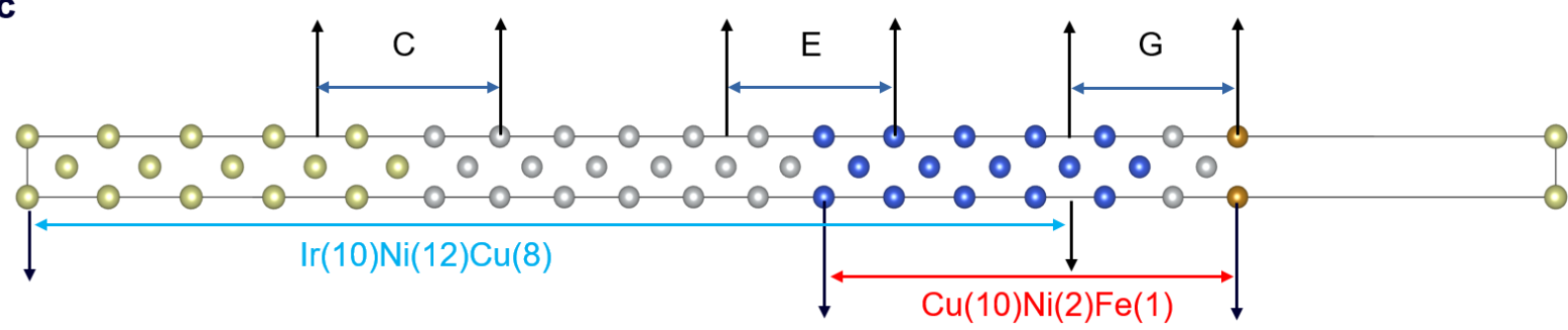

Figure S1. Definition and optimization of the material stack for DFT calculations. a Schematic of the materials stack used for carrying out the DFT calculations. b Interlayer distances at equilibrium, for the three different in-plane lattice constants here investigated. $\mathbf{c}$ Definition of the two sub-systems investigated for the calculations of the magnetic anisotropy in the reference and the free magnet. 
The materials stack in Fig S1a is simulated for three different conditions, where the in-plane lattice constants are derived from the bulk lattice constant of $\mathrm{Ir}, \mathrm{Cu}$ and Ni: $\mathrm{a}_{\mathrm{Ir}}=3.84 \AA$, $\mathrm{a}_{\mathrm{Ni}}=3.52 \AA, \mathrm{a}_{\mathrm{Cu}}=3.61 \AA$. Since $\mathrm{Ir}, \mathrm{Ni}$ and $\mathrm{Cu}$ are all fcc structures and we are interested in the stack growing along the [001] crystallographic direction, the primitive cell of (001) surface is a square lattice with in-plane lattice constants of $2.715 \AA, 2.489 \AA$ and $2.553 \AA$ for Ir, Ni and Cu, respectively. Accordingly, once the in-plane lattice constants are taken of a particular material, only the interlayer lattice constants are optimized (see Fig. S1). Due to the large thickness of the investigated materials stack, we decided to divide it into two sub-systems: $\operatorname{Ir}(10) \backslash \mathrm{Ni}(12) \backslash \mathrm{Cu}(8)$ and $\mathrm{Cu}(10) \backslash \mathrm{Ni}(2) \backslash \mathrm{Fe}(1-2)$, with thicknesses given in monolayers. Those two sub-stacks are used for the calculation of the magnetic properties of the two magnets: the Ni(12) reference magnet and the $\mathrm{Ni}(2) \backslash \mathrm{Fe}(1-2)$ free magnet. Note, we have tested using the full multilayers with $t_{\mathrm{Cu}}=6$ that the MCA does not change sign and magnitude significantly. After getting the out-of-plane relaxed structure of $\operatorname{Ir}(10) \backslash \mathrm{Ni}(12) \backslash \mathrm{Cu}(8)$ and $\mathrm{Cu}(10) \backslash \mathrm{Ni}(2) \backslash \mathrm{Fe}(1)$ (see Fig. S1c), the Ir-Ni (C) and $\mathrm{Ni}-\mathrm{Cu}(\mathrm{E})$, and Cu-Ni-Fe (G) interfacial forces are relaxed below $1 \mathrm{meV} / \mathrm{A}$. It is important to mention that the two sub-stacks are optimized separately. The layers B, D, E (see Fig S1a) are treated as unstrained interlayer distances after the optimization.

Tables in Figs. S2a and b report the calculated spin and orbital magnetic moment at both interfaces in the Ir $\backslash \mathrm{Ni} \backslash \mathrm{Cu}$ and $\mathrm{Cu} \backslash \mathrm{Ni} \backslash$ Fe sub-systems, respectively. On the other hand, Table in Fig. S2c shows the calculated magneto-crystalline anisotropies in the two sub-geometries. The bottom magnet is predicted to have a perpendicular magnetic anisotropy (PMA) for the layer thicknesses here investigated, regardless of the

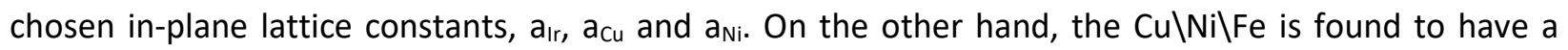
magnetic anisotropy which depends on the chosen in-plane lattice constant and the thickness of the Fe layer. Note, the anisotropy values are well converged for $32 \times 32 \mathrm{k}$-pint mesh in the 2D-BZ. The reported MCA values were obtained for $48 \times 48$ k-point grid in the $2 D-B Z$. 
a Ni bulk

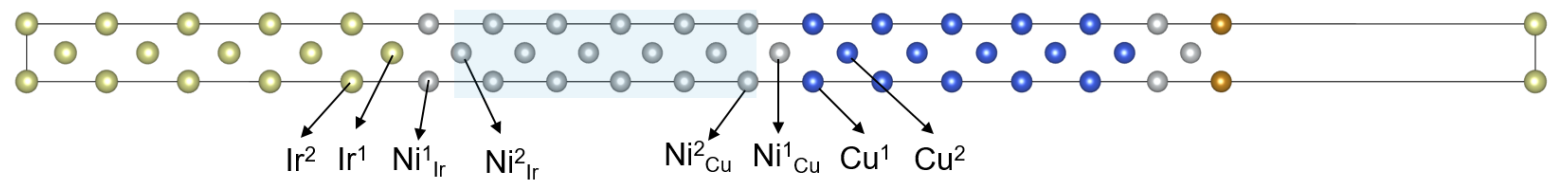

\begin{tabular}{|c|c|c|c|c|c|c|}
\hline $\begin{array}{c}\text { Posi- } \\
\text { tion }\end{array}$ & $\begin{array}{c}\text { Mag. } \\
\text { Mom. } \\
\left(\mathrm{a}_{\mathrm{lr}}\right)\end{array}$ & $\begin{array}{c}\text { Orb. } \\
\text { Mom. }\end{array}$ & $\begin{array}{c}\text { Mag. } \\
\text { Mom. } \\
\left(\mathrm{a}_{\mathrm{Cu}}\right)\end{array}$ & $\begin{array}{c}\text { Orb. } \\
\text { Mom. }\end{array}$ & $\begin{array}{c}\text { Mag. } \\
\text { Mom. } \\
\left(\mathrm{a}_{\mathrm{Ni}}\right)\end{array}$ & $\begin{array}{c}\text { Orb. } \\
\text { Mom. }\end{array}$ \\
\hline $\mathbf{I r}^{2}$ & -0.023 & -0.003 & 0.00 & -0.001 & 0.023 & 0.0 \\
\hline $\mathbf{I r}^{1}$ & 0.062 & 0.01 & 0.11 & 0.013 & 0.14 & 0.014 \\
\hline $\mathbf{N i}^{1}{ }_{\text {Ir }}$ & 0.28 & 0.02 & 0.44 & 0.032 & 0.51 & 0.032 \\
\hline $\mathbf{N i}^{2}{ }_{\text {Ir }}$ & 0.58 & 0.057 & 0.65 & 0.057 & 0.65 & 0.054 \\
\hline $\mathbf{N i}_{\text {bulk }}$ & $\sim 0.6$ & & $\sim 0.65$ & & $\sim 0.65$ & \\
\hline
\end{tabular}

\begin{tabular}{|c|c|c|c|c|c|c|}
\hline $\begin{array}{c}\text { Posi- } \\
\text { tion }\end{array}$ & $\begin{array}{c}\text { Mag. } \\
\text { Mom. } \\
\left(\mathbf{a}_{\text {Ir }}\right)\end{array}$ & $\begin{array}{c}\text { Orb. } \\
\text { Mom. }\end{array}$ & $\begin{array}{c}\text { Mag. } \\
\text { Mom. } \\
\left(\mathbf{a}_{\mathrm{Cu}}\right)\end{array}$ & $\begin{array}{c}\text { Orb. } \\
\text { Mom. }\end{array}$ & $\begin{array}{c}\text { Mag. } \\
\text { Mom. } \\
\left(\mathbf{a}_{\mathrm{Ni}}\right)\end{array}$ & $\begin{array}{c}\text { Orb. } \\
\text { Mom. }\end{array}$ \\
\hline $\mathrm{Cu}^{2}$ & -0.003 & 0.000 & -0.004 & 0.0 & -0.004 & 0.0 \\
\hline $\mathrm{Cu}^{1}$ & -0.003 & 0.000 & -0.005 & 0.0 & -0.006 & 0.0 \\
\hline $\mathrm{Ni}^{1} \mathrm{Cu}$ & 0.41 & 0.04 & 0.46 & 0.044 & 0.45 & 0.04 \\
\hline $\mathrm{Ni}^{2} \mathrm{Cu}$ & 0.59 & 0.052 & 0.63 & 0.055 & 0.62 & 0.047 \\
\hline
\end{tabular}

b

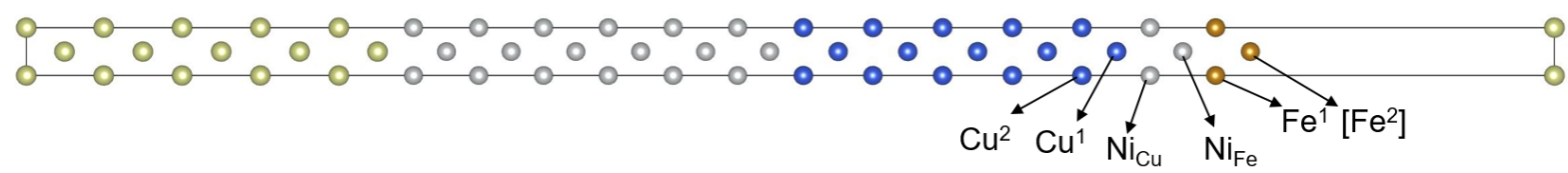

\begin{tabular}{|c|c|c|c|c|c|c|}
\hline Position & $\begin{array}{c}\text { Mag. Mom. } \\
\left(a_{1 r}\right) \\
{[y=2]}\end{array}$ & $\begin{array}{l}\text { Orb. Mom. } \\
{[y=2]}\end{array}$ & $\begin{array}{c}\text { Mag. Mom. } \\
\left(a_{c u}\right) \\
{[y=2]}\end{array}$ & $\begin{array}{l}\text { Orb. Mom. } \\
\quad[y=2]\end{array}$ & $\begin{array}{c}\text { Mag. Mom. } \\
\left(a_{\text {Ni }}\right) \\
{[y=2]}\end{array}$ & $\begin{array}{c}\text { Orb. Mom. } \\
{[y=2]}\end{array}$ \\
\hline $\mathrm{Cu}^{2}$ & $\begin{array}{c}-0.003 \\
{[-0.004]}\end{array}$ & $\begin{array}{c}0.0 \\
{[0.0]}\end{array}$ & $\begin{array}{c}-0.002 \\
{[-0.004]}\end{array}$ & $\begin{array}{c}0.0 \\
{[0.0]}\end{array}$ & $\begin{array}{c}-0.003 \\
{[-0.004]}\end{array}$ & $\begin{array}{c}0.0 \\
{[0.0]}\end{array}$ \\
\hline $\mathrm{Cu}^{1}$ & $\begin{array}{c}-0.005 \\
{[-0.001]}\end{array}$ & $\begin{array}{c}0.0 \\
{[-0.001]}\end{array}$ & $\begin{array}{c}0.0 \\
{[-0.006]}\end{array}$ & $\begin{array}{c}0.0 \\
{[-0.001]}\end{array}$ & $\begin{array}{c}-0.002 \\
{[-0.003]}\end{array}$ & $\begin{array}{c}0.0 \\
{[0.0]}\end{array}$ \\
\hline $\mathbf{N i}_{\mathrm{Cu}}$ & $\begin{array}{c}0.26 \\
{[0.31]}\end{array}$ & $\begin{array}{c}0.039 \\
{[0.034]}\end{array}$ & $\begin{array}{c}0.34 \\
{[0.34]}\end{array}$ & $\begin{array}{c}0.34 \\
{[0.037]}\end{array}$ & $\begin{array}{c}0.33 \\
{[0.36]}\end{array}$ & $\begin{array}{c}0.03 \\
{[0.036]}\end{array}$ \\
\hline $\mathrm{Ni}_{\mathrm{Fe}}$ & $\begin{array}{c}0.61 \\
{[0.64]}\end{array}$ & $\begin{array}{c}0.058 \\
{[0.055]}\end{array}$ & $\begin{array}{c}0.62 \\
{[0.56]}\end{array}$ & $\begin{array}{c}0.036 \\
{[0.042]}\end{array}$ & $\begin{array}{c}0.58 \\
{[0.55]}\end{array}$ & $\begin{array}{c}0.034 \\
{[0.039]}\end{array}$ \\
\hline $\mathrm{Fe}^{1}$ & $\begin{array}{c}2.95 \\
{[2.62]}\end{array}$ & $\begin{array}{c}0.109 \\
{[0.064]}\end{array}$ & $\begin{array}{c}2.90 \\
{[2.62]}\end{array}$ & $\begin{array}{c}0.071 \\
{[0.069]}\end{array}$ & $\begin{array}{c}2.84 \\
{[2.60]}\end{array}$ & $\begin{array}{c}0.066 \\
{[0.064]}\end{array}$ \\
\hline$\left[\mathrm{Fe}^{2}\right]$ & [2.91] & [0.102] & [2.82] & {$[0.071]$} & [2.75] & [0.063] \\
\hline
\end{tabular}

C

\begin{tabular}{|c|c|c|c|}
\hline k-points & $\operatorname{MCA}\left(a_{\mid r}\right)$ & $\operatorname{MCA}\left(a_{c u}\right)$ & $\operatorname{MCA}\left(a_{N i}\right)$ \\
\hline $32 \times 32$ & 0.31 & 0.40 & 0.18 \\
\hline
\end{tabular}

\begin{tabular}{|c|c|c|c|}
\hline k-points & $\begin{array}{c}\text { MCA }\left(a_{1 r}\right) \\
{[y=2]}\end{array}$ & $\begin{array}{c}\text { MCA }\left(a_{c u}\right) \\
{[y=2]}\end{array}$ & $\begin{array}{c}\operatorname{MCA}\left(a_{N i}\right) \\
{[y=2]}\end{array}$ \\
\hline $32 \times 32$ & $\begin{array}{c}0.56 \\
{[0.028]}\end{array}$ & $\begin{array}{l}-0.074 \\
{[0.28]}\end{array}$ & $\begin{array}{l}-0.25 \\
{[0.14]}\end{array}$ \\
\hline $48 \times 48$ & $\begin{array}{c}0.55 \\
{[-0.008]}\end{array}$ & $\begin{array}{l}-0.098 \\
{[0.305]}\end{array}$ & $\begin{array}{l}-0.222 \\
{[0.154]}\end{array}$ \\
\hline
\end{tabular}

Figure S2. Calculated magnetic properties of the reference and free magnet. a Calculated spin and orbital magnetic moment at both interfaces in $\operatorname{Ir}(10) \backslash \mathrm{Ni}(12) \backslash \mathrm{Cu}(8)$. b Calculated spin and orbital magnetic moment at both interfaces in $\mathrm{Cu}(10) \backslash \mathrm{Ni}(2) \backslash \mathrm{Fe}(1-2)$. c Calculated magneto-crystalline anisotropies (MCA) in $\operatorname{Ir}(10) \backslash \mathrm{Ni}(12) \backslash \mathrm{Cu}(8)(\mathrm{left})$ and $\mathrm{Cu}(10) \backslash \mathrm{Ni}(2) \backslash \mathrm{Fe}(1-2)$ (right). MCA > (<) 0 indicates that the magnetic moments are stable along the out-of-plane (in-plane) direction. 
Due to the large distance between the Ir buffer and the top magnet, in reality the Ni\Fe bilayer is expected to have an in-plane lattice constant which is close to that of the thick $\mathrm{Cu}$ spacer. Based on this, the free magnet is expected to show an in-plane magnetic anisotropy (MCA $<0)$ for $t_{\mathrm{Fe}}=1 \mathrm{ML}$ and a PMA (MCA > 0 ) for $t_{\mathrm{Fe}}=2 \mathrm{ML}$, which is what is actually observed experimentally, as discussed in the main text.

Finally, the interlayer exchange coupling in the whole stack (see Fig. S3a) is investigated. The interlayer exchange coupling is computed for materials stacks with both the $\mathrm{Cu}$ and the $\mathrm{Ni}$ in-plane lattice constant (left and right in Fig. S3b, respectively). The coupling and the corresponding effective magnetic field ( $\mathbf{B}_{\text {eff }}$ ) acting on the free magnet are calculated with and without spin-orbit coupling (SOC). $\mathbf{B}_{\text {eff }}>(<) 0$ indicates a ferromagnetic (anti-ferromagnetic) coupling between the reference magnet and the free magnet.

a

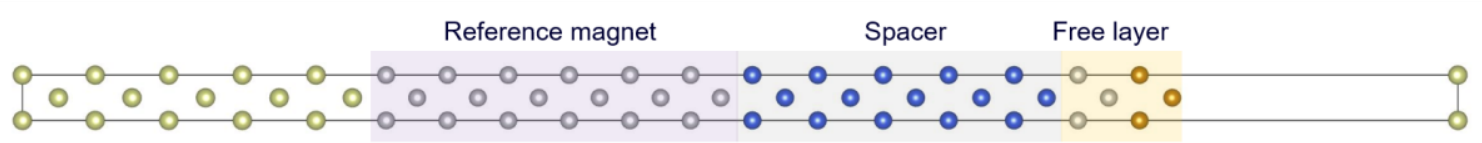

b

Cu lattice constant $\left(\mathrm{a}_{\mathrm{Cu}}=3.61 \AA\right)$

C

Ni lattice constant $\left(a_{\mathrm{Ni}}=3.52 \AA\right)$

\begin{tabular}{|c|c|c|c|c|}
\hline $\begin{array}{c}\mathrm{Cu} \\
\text { spacer } \\
\text { thickness } \\
\text { (ML) }\end{array}$ & $\begin{array}{c}0.5^{\star}\left(\mathrm{E}_{\mathrm{afm}}-\mathrm{E}_{\mathrm{fm}}\right) \\
\text { w/o SOC } \\
(\mathrm{meV})\end{array}$ & $\begin{array}{c}B_{\text {eff }} \\
\text { w/o SOC } \\
\text { (T) }\end{array}$ & $\begin{array}{c}0.5^{*}\left(E_{\mathrm{afm}}-E_{\mathrm{fm}}\right) \\
\text { SOC } \\
(\mathrm{meV})\end{array}$ & $\begin{array}{c}\text { Beff }_{\text {eft }} \\
\text { SOC } \\
\text { (T) }\end{array}$ \\
\hline 8 & -0.1418 & -0.388 & -0.1283 & -0.351 \\
\hline 10 & -0.4862 & -1.329 & -0.1158 & -0.317 \\
\hline 12 & -0.4307 & -1.177 & -0.1810 & -0.495 \\
\hline 14 & -0.5643 & -1.542 & -0.0476 & -0.13 \\
\hline 16 & -0.2823 & -0.783 & 0.064 & 0.175 \\
\hline 18 & -0.1812 & -0.495 & 0.234 & 0.64 \\
\hline
\end{tabular}

\begin{tabular}{|c|c|c|c|c|}
\hline $\begin{array}{c}\text { Cu } \\
\text { spacer } \\
\text { thickness } \\
\text { (ML) }\end{array}$ & $\begin{array}{c}0.5^{*}\left(\mathrm{E}_{\mathrm{afm}}-\mathrm{E}_{\mathrm{fm}}\right) \\
\text { w/o SOC } \\
(\mathrm{meV})\end{array}$ & $\begin{array}{c}B_{\text {eff }} \\
\text { w/o SOC } \\
\text { (T) }\end{array}$ & $\begin{array}{c}0.5^{\star}\left(E_{\mathrm{afm}}-\mathrm{E}_{\mathrm{fm}}\right) \\
\mathrm{SOC} \\
(\mathrm{meV})\end{array}$ & $\begin{array}{c}B_{\text {eff }} \\
\text { SOC } \\
\text { (T) }\end{array}$ \\
\hline 8 & -0.1122 & -0.31 & 0.0726 & 0.2 \\
\hline 10 & -0.0922 & -0.255 & 0.2160 & 0.597 \\
\hline 12 & -0.5605 & -1.549 & -0.4163 & -1.15 \\
\hline 14 & -0.4604 & -1.272 & -0.2004 & -0.554 \\
\hline
\end{tabular}

$1 \mu_{\mathrm{B}}=0.0579 \mathrm{meV} / \mathrm{Tesla}, \mathrm{S}_{\text {free-layer }}=6.32 \mu_{\mathrm{B}}$

Figure S3. Calculated interlayer exchange coupling between the reference and free magnet. a Materials stack used for the calculation of the interlayer exchange coupling. b,c Calculated interlayer exchange coupling and corresponding effective magnetic field acting on the free magnet, as a function of the space layer thickness. In panel $b$ (c) the calculated values for the stack with the $\mathrm{Cu}(\mathrm{Ni})$ in-plane lattice constant are provided, respectively.

\section{FLEUR}

Additionally, in order to understand the magnetic structure of the free layer, DFT calculations using the local density approximation (LDA) to the exchange correlation functional were employed using the full potential linearized augmented plane wave (FLAPW) method as implemented in the FLEUR code ${ }^{8,9}$. We have used the non-collinear version of the code because of the implementation of treating magnetic multilayer with more than one magnetic atom in the unit-cell. We have used the experimental Cu bulk 
lattice constant of $3.61 \AA$ that corresponds to the in-plane lattice constant of $2.553 \AA$. The free magnetic layer was modelled by an asymmetric film geometry of $\mathrm{Cu}(100) \backslash \mathrm{Ni}(2) \backslash \mathrm{Fe}(2)$ with fcc stacking where six layers of $\mathrm{Cu}$ were used as substrate. The MCA energy was further calculated using force theorem starting from the converged charge density of collinear magnetic state. Note, the MCA was found to be 0.337 meV, little higher than the VASP value, that is, $0.305 \mathrm{meV}$. The energy of spin spiral (SS) characterized by wave vector $\mathbf{q}$, was calculated using the generalized Bloch theorem ${ }^{10}$. The $\mathbf{q}$-vectors were taken along the high-symmetry direction of the 2D-BZ. The Dzyaloshinskii-Moriya interaction (DMI) was calculated from the SOC contribution within first-order perturbation theory ${ }^{11}$ in the vicinity of SS ground state which was obtained without SOC. The magnetic force theorem was employed to determine the SS states with and without $\mathrm{SOC}^{12}$. We considered $240 \times 240 \mathrm{k}^{\prime \prime}$-grid points in the $2 \mathrm{D}-\mathrm{BZ}$ and a cut-off of $\mathrm{k}_{\max }=4.0 \mathrm{a}^{-\mathrm{u}^{-1}}$ to expand the LAPW basis functions. The magnetic layer decomposed SS energies (top Fe(2) and interface $\mathrm{Ni}(2)$ layers) were calculated in such a way that the magnetic moments of the considered layer with flat SS were confined in the plane while the rest of the magnetic moments were assumed perpendicular to the plane. Therefore, the interlayer coupling between layers became zero while intralayer coupling in the considered layer can be calculated from the SS energy dispersions. The interlayer exchange coupling was calculated by fitting the energy as a function of the angle between two magnetic layers i.e. by varying the angle between $\mathrm{Fe}(2)$ and $\mathrm{Ni}(2)$ magnetizations simply in a noncollinear framework. The angular dependent energies were fitted with the spin Hamiltonian, $\mathrm{H}_{\mathrm{EX}}=-\sum_{\mathrm{nn}} \mathrm{J}_{\mathrm{nn}}\left(\mathbf{m}_{\mathrm{i}} \cdot \mathbf{m}_{\mathrm{j}}\right)$, where $\mathbf{m}_{\mathrm{i}}$ is the unit vector, defining the orientation of spin. $J_{n n}$ in the Hamiltonian is the effective nearest neighbour exchange constant i.e. $\mathrm{J}^{\mathrm{FF}}, \mathrm{J}^{\mathrm{NN}}, \mathrm{J}^{\mathrm{FN}}$ and $\mathrm{J}_{\text {eff }}$ in the main text. The layer decomposed SOC contribution in the SS states were modelled with the spin Hamiltonian $\mathrm{H}_{\mathrm{DM}}=-\sum_{\mathrm{nn}} \mathbf{D}_{\mathrm{nn}} \cdot\left(\mathbf{m}_{\mathrm{i}} \times \mathbf{m}_{\mathrm{j}}\right)$, where $\mathbf{D}_{\mathrm{nn}}$ is the nearest neighbour DMI vector around an atom. The (001) oriented fcc film exhibits a $\mathrm{C}_{4 v}$ symmetry, therefore, the $\mathbf{D}_{\mathrm{nn}}$ is perpendicular to the bond connecting nearest neighbour atoms. Such DMI contributions always support Nèel type chiral domain or SS state. The $D_{n n}$ in the model is equivalent to $D^{\mathrm{FF}}, D^{\mathrm{NN}}, D_{\text {eff }}$ in the main text. It is worth to mention that two SS states in two magnetic layers are assumed to be connected by the interlayer exchange coupling and the effective exchange model with intralayer and interlayer couplings is in good agreement with the dispersion energy $\mathrm{E}(\mathbf{q})$ for the full system close to the ferromagnetic state, see the green symbols and the fitted curve in Fig. 2(a) in the main text. 


\section{S2. Growth of epitaxial Ir buffer layers on $\mathrm{MgO}$ single crystals}

$\mathrm{MgO}(001)$ and $\mathrm{MgO}(111)$ substrates were cleaned by repeated flash annealing at $1,300 \mathrm{~K}$ in a $3.0 \times 10^{-8} \mathrm{Torr}_{2}$ atmosphere and subsequent Oxygen-Argon plasma cleaning. The clean $\mathrm{MgO}$ surface was confirmed by Auger electron spectroscopy and LEEM imaging. Furthermore, high-quality low-energy electron diffraction (LEED) patterns were obtained, indicating a well-ordered surface.

The Ir buffer layer is deposited by physical vapor deposition from an electron beam evaporator in UHV conditions, at a pressure of about $5.0 \times 10^{-9}$ Torr. On top of the $\mathrm{MgO}(001)$ substrates, the Ir buffer layer is deposited with the substrates at a temperature of $770 \mathrm{~K}$. After the deposition, in order to obtain a well ordered $\operatorname{Ir}(001)-(1 \times 1)$ surface, one flash annealing at $1120 \mathrm{~K}$ in a $5.0 \times 10^{-6}$ Torr $\mathrm{O}_{2}$ atmosphere followed by a 5 min annealing at about $400 \mathrm{~K}$ in a $2.0 \times 10^{-7}$ Torr $\mathrm{H}_{2}$ atmosphere were carried out ${ }^{13}$. The $\operatorname{Ir}(001)-(1 \times 1)$

surface state was confirmed by LEED (see Fig. S.4). On the $\mathrm{MgO}(111)$ substrates, depositing Ir at substrate's temperature of $770 \mathrm{~K}$ was sufficient to obtain a well ordered $\operatorname{Ir}(111)$ surface, as confirmed by LEED. 
a
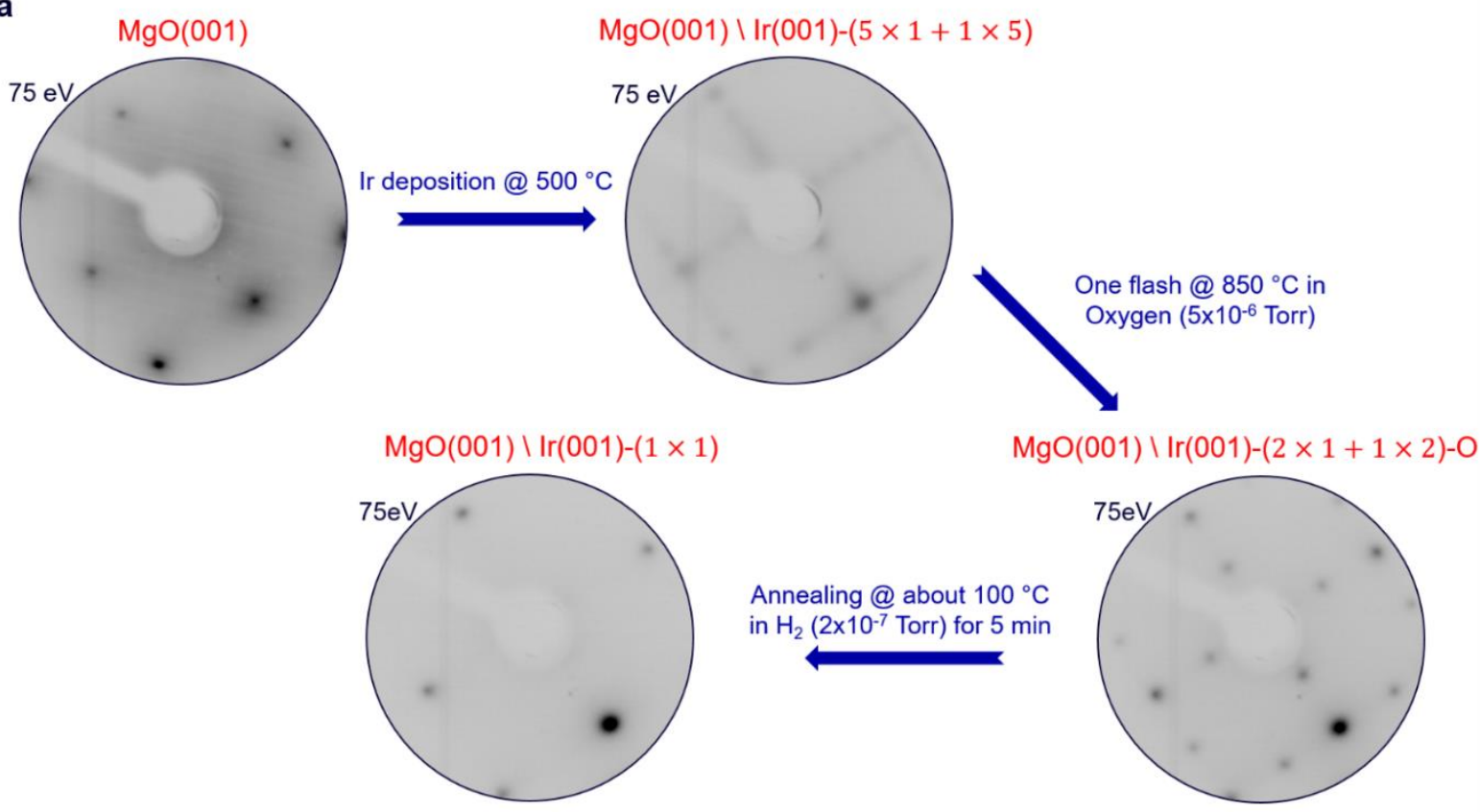

b
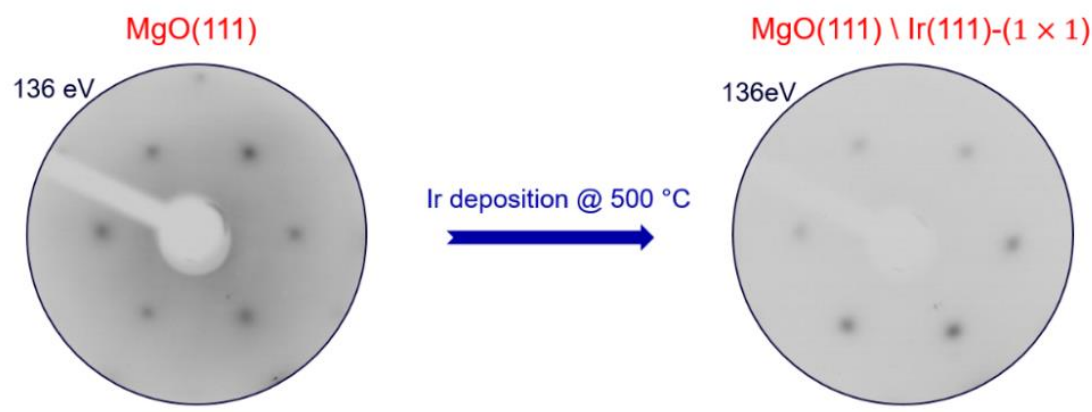

Figure S4. Growth of epitaxial Ir buffer layers on top of MgO single crystals. a Preparation of an epitaxial $\operatorname{Ir}(001)$ buffer layer on top of an $\mathrm{MgO}(001)$ single crystal. An appropriate process of growth and annealing was developed to successfully stabilize an $\operatorname{Ir}(001)-(1 \times 1)$ surface needed for the room temperature growth of the desired epitaxial magnetic multilayer, as shown by the reported low energy electron diffraction (LEED) patterns. The $\operatorname{Ir}(001)$ surface stabilization process developed by Z. Tian et $a l .{ }^{13}$ was taken as a guidance. All LEED patterns were acquired at an electron-beam energy of $75 \mathrm{eV}$. b Preparation of an epitaxial $\operatorname{Ir}(111)$ buffer layer on top of an MgO(111) single crystal. In this case a simple growth of the heavy metal on a substrate at $500{ }^{\circ} \mathrm{C}$ was enough to have the required (111) unreconstructed surface needed for the room temperature growth of the desired epitaxial magnetic multilayer. All LEED patterns were acquired at an electron-beam energy of $136 \mathrm{eV}$. 


\section{S3. Growth calibration by LEEM}

a

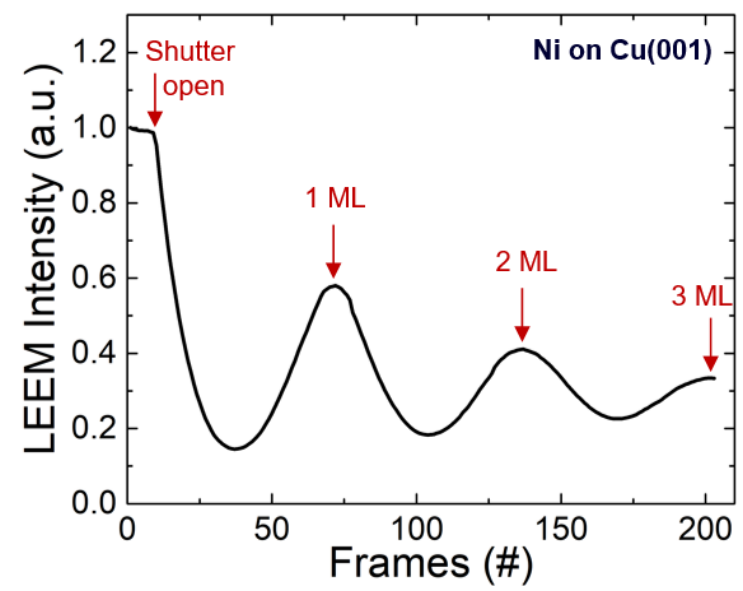

b

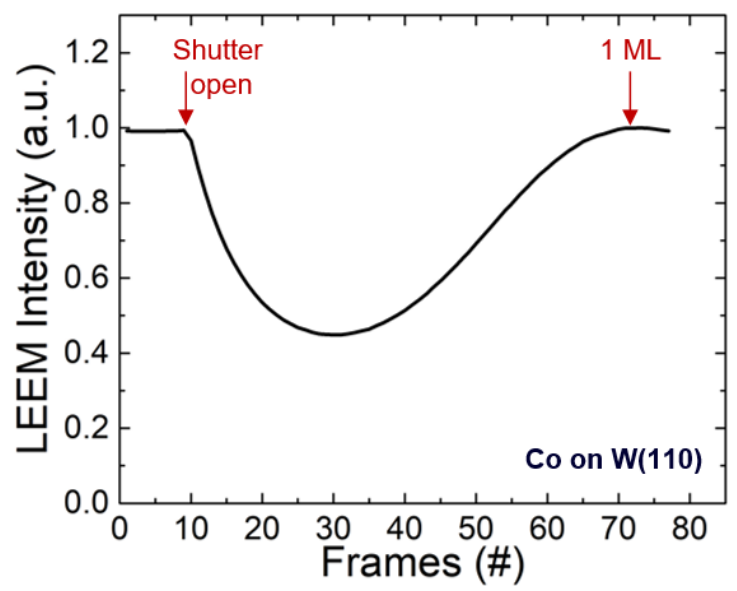

Figure S5. Materials growth calibration via LEEM. The growth rate of the deposited materials was calibrated by monitoring the low-energy electron microscope (LEEM) intensity oscillations. The dependency of the intensity of the reflected beam on the substrate coverage allows for the monitoring of the layer-by-layer growth of the deposited material ${ }^{14}$. As examples of the described growth calibration method, $\mathbf{a}$ and $\mathbf{b}$ report the growth of $\mathrm{Ni}$ on a $\mathrm{Cu}(001)$ single crystal and of Co on a W(110) single crystal, respectively. 


\section{S4. Protocol for sample preparation}

All the materials stacks were grown on top of the Ir buffer layer in-situ, inside the SPLEEM chamber, under UHV conditions with a base pressure of about $5.0 \times 10^{-10}$ Torr or below. All the materials $(\mathrm{Fe}, \mathrm{Co}, \mathrm{Ni}, \mathrm{Cu}$ and Ir) were deposited by physical vapor deposition from electron beam evaporators with the substrate at room temperature. The epitaxial character of the growth was confirmed by the strong electron reflectivity observed in the LEEM images. The growth rates for the depositions on the $\mathrm{MgO}(001)$ substrates were calibrated using a $\mathrm{Cu}(001)$ crystal, while the growth rates for depositions on the $\mathrm{MgO}(111)$ substrates were calibrated using a W(110) crystal. The wedged Cu spacer was deposited via a shadow mask approach in a different vacuum chamber directly connected to the SPLEEM chamber.
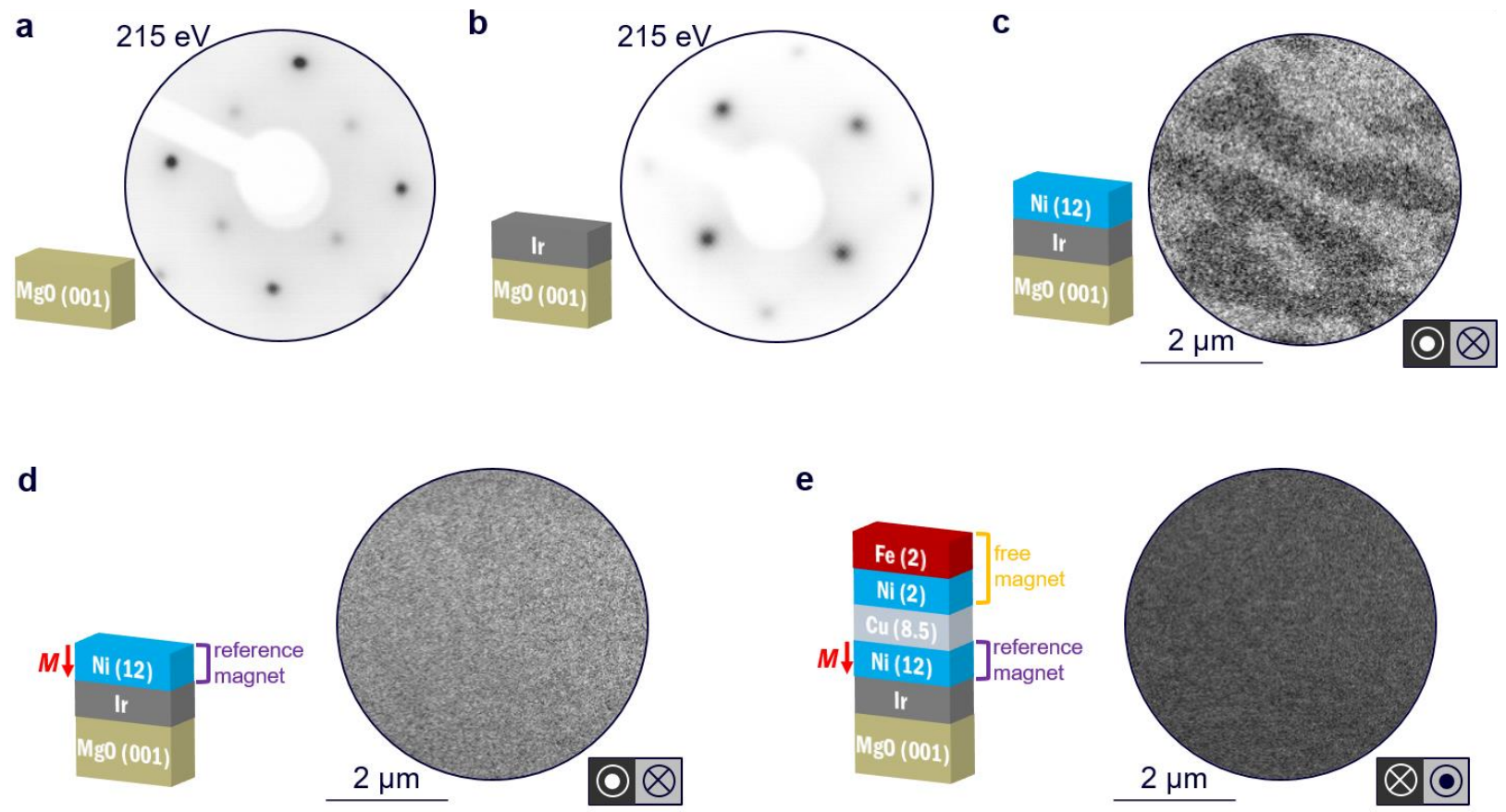

Figure S6. Sample preparation protocol on $\mathrm{MgO}(001)$ substrates. Here, the preparation of a sample on a $\mathrm{MgO}(001)$ single crystal is described. a LEED pattern from the top surface of the bare $\mathrm{MgO}(001)$ crystal. b LEED pattern of the deposited Ir buffer layer, showing the successfully stabilized $\operatorname{Ir}(001)-(1 \times 1)$ surface. Both LEED patterns were acquired at an electron-beam energy of $215 \mathrm{eV}$. c SPLEEM image showing the OOP magnetic domains pattern in the bottom $\mathrm{Ni}(12 \mathrm{ML})$ layer. d SPLEEM image showing the OOP single domain state in the reference magnet obtained by applying, in an adjacent vacuum chamber, a saturating magnetic field of $-500 \mathrm{mT}$ along the OOP direction. The reference magnet is now in a single domain state with the magnetization pointing down (-z direction). e SPLEEM image showing the OOP magnetic domain pattern in the free magnetic layer. A single domain state is observed for a $\mathrm{Cu}$-spacer thickness of 8.5 ML. 

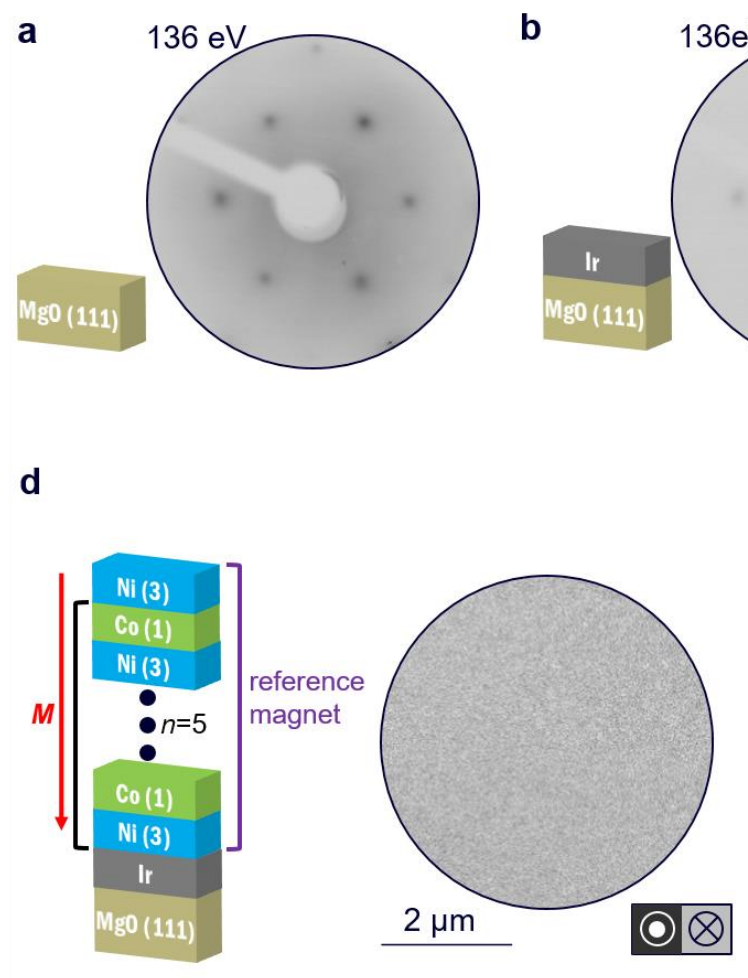
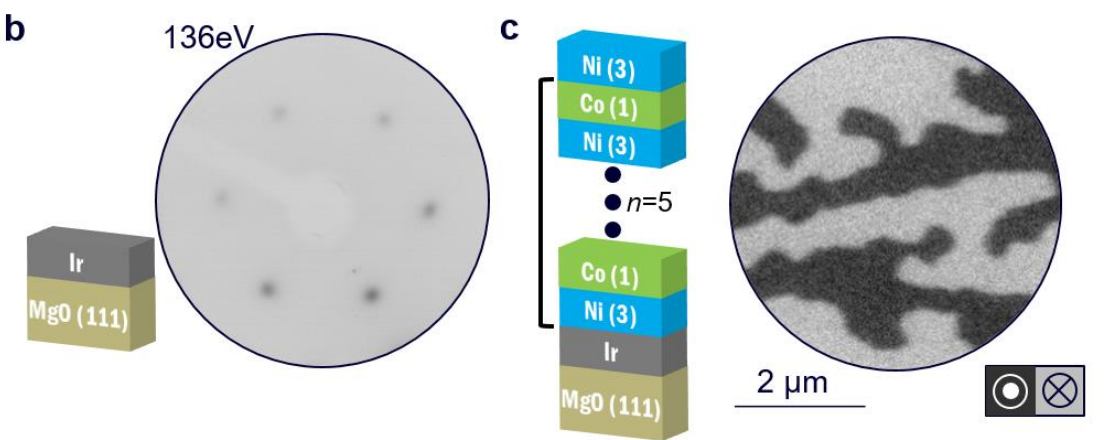

e

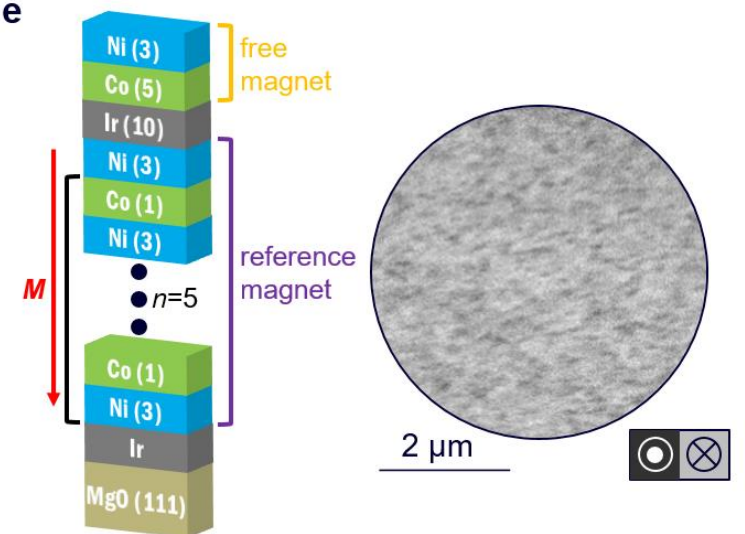

Figure S7. Sample preparation protocol on $\mathrm{MgO}(111)$ substrates. Here, the preparation of a sample on a $\mathrm{MgO}(111)$ single crystal is described. a LEED pattern from the top surface of the bare $\mathrm{MgO}(111)$ crystal. b LEED pattern of the deposited Ir buffer layer, showing the successfully stabilized $\operatorname{Ir}(111)-(1 \times 1)$ surface. Both LEED patterns were acquired at an electron-beam energy of $136 \mathrm{eV}$. c SPLEEM image showing the OOP magnetic domains pattern in the bottom [ $\mathrm{Ni}(3 \mathrm{ML}) \backslash \mathrm{Co}(1 \mathrm{ML})]_{5} \backslash \mathrm{Ni}(3 \mathrm{ML})$ multilayer. d SPLEEM image showing the OOP single domain state in the reference magnet obtained by applying, in an adjacent vacuum chamber, a saturating magnetic field of $-500 \mathrm{mT}$ along the OOP direction. The reference magnet is now in a single domain state with magnetization pointing down (-z direction). e SPLEEM image showing the OOP magnetic domain pattern in the free magnetic layer. Small magnetic bubbles (dark spots) are observed for an Ir-spacer thickness of $10 \mathrm{ML}$. 


\section{S5. Angle distribution of the domain walls spin-texture}

a

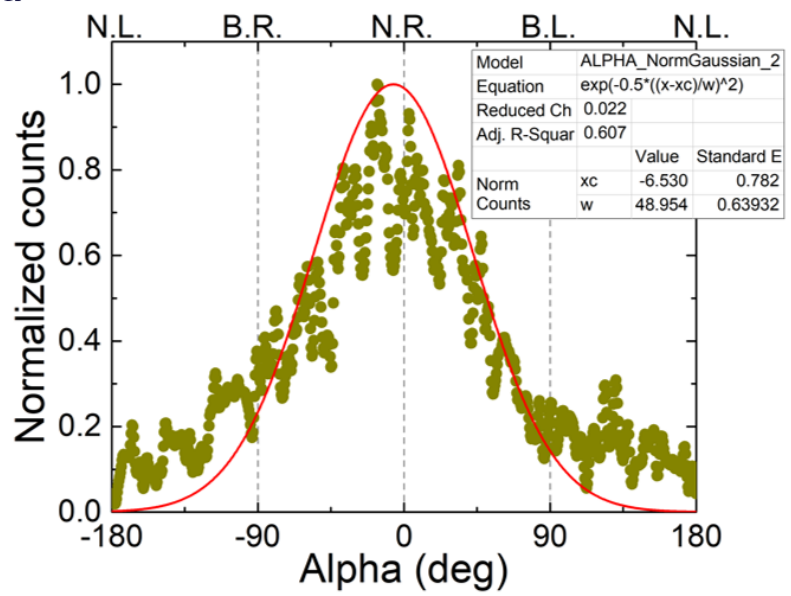

b

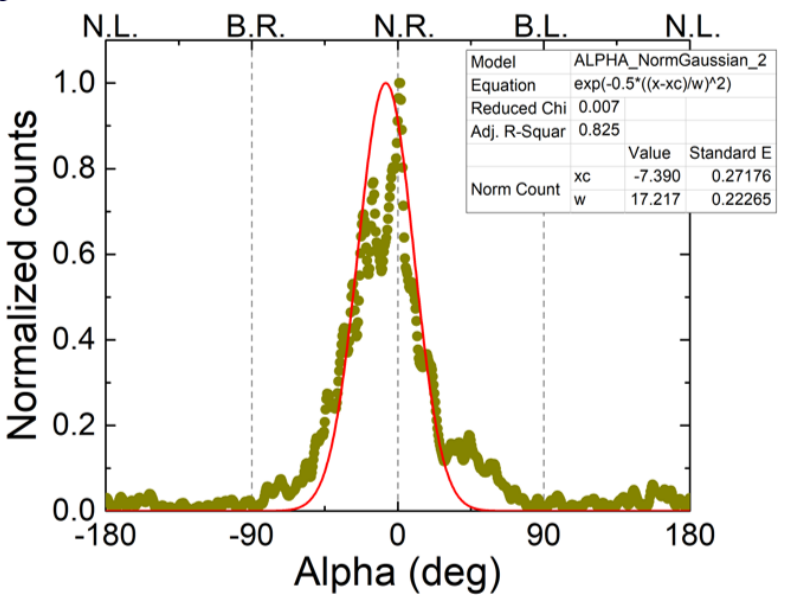

Figure S8. Magnetic domain wall's angle distributions. Here, the angle distributions of the DW spin-textures for the two investigated free magnets are shown. a Histogram of the DW magnetization angle distribution (gold dots) for the $\mathrm{Cu}(001) \backslash \mathrm{Ni}(2) \backslash \mathrm{Fe}(2)$ system (as reported in Fig. $3 \mathrm{c}$ of the main text). The red solid line is the Gaussian fit curve to the experimental data. As shown by the fitting parameters, the full width of the fitting curve is $2 \mathrm{w}=98.0^{\circ} \pm 0.8^{\circ}$. b Histogram of the DW magnetization angle distribution (gold dots) for the $\operatorname{Ir}(111) \backslash \mathrm{Co}(5) \backslash \mathrm{Ni}(3)$ system (as reported in Fig. 4h of the main text). The red solid line is the Gaussian fit curve to the experimental data. As shown by the fitting parameters, the width of the fitting curve is $2 \mathrm{w}=34.4^{\circ} \pm 0.3^{\circ}$. The values in parentheses indicate the magnetic layers' thicknesses in monolayers (ML). 


\section{S6. Imaging of the 3D spin-texture via spin-polirized low energy electron microscopy (SPLEEM)}

Real-space magnetic imaging. The real-space magnetic images were acquired using the SPLEEM instrument at the National Center for Electron Microscopy at the Lawrence Berkeley National Laboratory $^{15}$. In SPLEEM images, the contrast in each pixel is obtained by calculating the asymmetry of the spin-dependent reflection between spin-up and spin-down electron beams, which is $A=\left(I_{\uparrow}-I_{\downarrow}\right) /\left(I_{\uparrow}+\right.$ $I_{\downarrow}$ ). This asymmetry $A$ is proportional to $\mathbf{P} \cdot \mathbf{M}$, where $\mathbf{P}$ is the spin polarization vector of the electron beam and $\mathbf{M}$ is the magnetization vector. The Cartesian components $M_{\mathrm{x}}, M_{\mathrm{y}}$ and $M_{\mathrm{z}}$ of the magnetization were resolved by taking sets of images with the electron beam spin polarization aligned along the $x, y, z$ directions, respectively ${ }^{16}$. All images were measured on samples held at room temperature. The energy of the incident electron beam was carefully chosen for each system in order to optimize magnetic contrast.

Vector field analysis. The method to analyse DWs chirality from the SPLEEM images was described by G. Chen et al. ${ }^{16}$. The DW normal vector $\mathbf{n}$ is determined from out-of-plane SPLEEM images, and the DW magnetization vector $\mathbf{m}$ is extracted from the asymmetry values of two in-plane SPLEEM images with orthogonal spin-polarizations. 


\section{Section S7. Stability of IEC-stabilized ferromagnetic skyrmions against an external magnetic field}

We imaged the magnetic domain state of the free layer of a sample made of $\mathrm{MgO}(001) \backslash \mathrm{Ir} \backslash \mathrm{Ni}(12) \backslash \mathrm{Cu}(7) \backslash \mathrm{Ni}(2) \backslash \mathrm{Fe}(2)$ after we applied a saturating magnetic field of $-0.5 \mathrm{~T}$ and $+0.5 \mathrm{~T}$ along the out-of-plane $(z)$ direction in an ultra-high vacuum chamber directly connected to our microscope. The relevant results are reported in Fig. S9. As it can be seen in the two images reporting the magnetic state after applying a $-0.5 \mathrm{~T}(\mathrm{a})$ and $+0.5 \mathrm{~T}(\mathrm{~b})$ external magnetic field, magnetic skyrmions are observed in the remanent states with core magnetization pointing antiparallel to the applied magnetic field. This demonstrates that the IEC-stabilized skyrmions are robust against the application of an external magnetic field.

It is worth mentioning that, even if we are not able to observe the magnetic state of the sample while applying the external field, the magnetic skyrmions observed after such process can be interpreted as the result of two different and complementary effects. 1 . The magnetic state of the free layer is completely saturated during the application of the external $0.5 \mathrm{~T}$ magnetic field and once the field is removed new skyrmions are nucleated due to the presence of the IEC effective field. 2. Not all the magnetic features present in the free layer before the application of the external field are annihilated by the field, and after removing the field they act as nucleation points for some of the skyrmions visible in the imaged in Fig. S9. The latter is a process well explained by J. E. Davies and co-workers ${ }^{17}$. Those two effects are completely independent one from the other and could together generate the final magnetic state shown in Fig. S9.

a

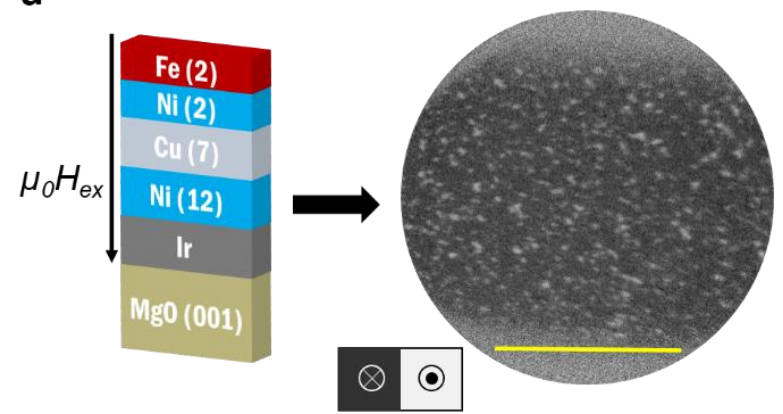

b

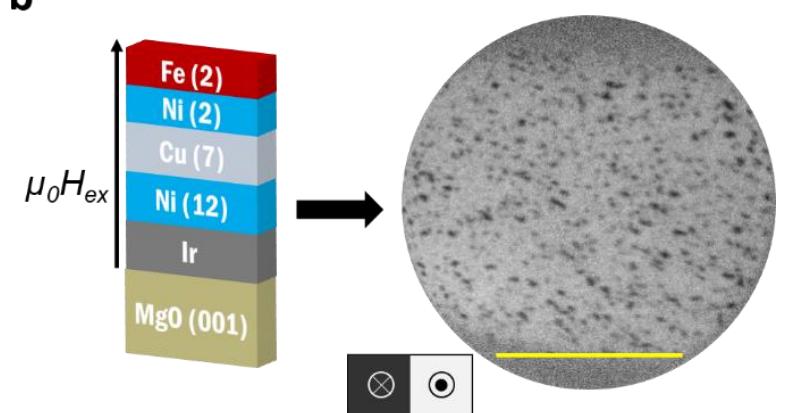

Figure S9. Robustness of zero-field magnetic skyrmions to an external magnetic field. a The remanent magnetic state of the free layer of a sample made of $\mathrm{MgO}(001) \backslash \mathrm{Ir} \backslash \mathrm{Ni}(12) \backslash \mathrm{Cu}(7) \backslash \mathrm{Ni}(2) \backslash \mathrm{Fe}(2)$, thicknesses in $\mathrm{ML}$, after the application of a saturating external magnetic field of $-0.5 \mathrm{~T}$ along the out-of-plane direction. $\mathbf{b}$ The remanent state of the same sample after the application of an external magnetic field of $+0.5 \mathrm{~T}$. In both cases the remanent state shows the presence of a magnetic background aligned parallel to the previously applied field and magnetic skyrmions with magnetization anti-aligned to it. The yellow scale bars indicate $5 \mu \mathrm{m}$. 


\section{S8. Investigation of the interlayer exchange coupling in $\mathrm{Cu}(001) \backslash \mathrm{Ni} \backslash \mathrm{Cu}(\mathrm{t}) \backslash \mathrm{Ni} \backslash \mathrm{Fe}$}

The interlayer magnetic coupling in the materials stack $\mathrm{Cu}(\mathbf{0 0 1}) \backslash \mathbf{N i}(\mathbf{1 2}) \backslash \mathrm{Cu}\left(\mathrm{t}_{\mathrm{cu}}\right) \backslash \mathrm{Ni}(\mathbf{2}) \backslash \mathrm{Fe}(\mathbf{2 . 5})$, all thicknesses in monolayers, was investigated. The two main differences between this sample and the samples discussed in the main manuscript are: 1 . the multilayer is grown on a $\mathrm{Cu}(001)$ single crystal, which helped obtaining an epitaxial growth with higher quality; 2 . the sample is free of the Ir buffer layer (not needed here since the PMA bottom Ni layer can be grown directly on the Cu substrate, while with the MgO substrate an Ir buffer layer is needed in order to promote epitaxy and out-of-plane anisotropy). The coupling between the reference and the free magnets is extracted in the same way as done for the wedge sample presented in our main manuscript. First, the reference layer is magnetized along the $+z$ direction, so to be in a single domain state. Second, after the deposition of the free layer, its magnetic state is imaged at different positions along the $\mathrm{Cu}$-wedge. In the graph of Fig. S10 we report, as a function of the $\mathrm{Cu}$ thickness, the normalized difference between the fractional areas of ferromagnetically (FM) and antiferromagnetically (AFM) coupled domains from a $10 \mu \mathrm{m}$ field of view.

As reported in Fig. S10, the coupling between the two magnetic layers is observed to change its sign as a function of the spacer thickness, oscillating between positive and negative coupling. This clearly demonstrates that the Ni\Cu\Ni\Fe stack is indeed characterized by an RKKY-type IEC between the two magnetic layers. Furthermore, the average oscillation period we can extract from the graph in Fig. S10 is about $7 \mathrm{ML}$, which is in good agreement with the theoretically predicted (6 ML) and experimentally observed $(6-8 \mathrm{ML})$ length of the long-period oscillation of RKKY coupling through a $\mathrm{Cu}(001)$ spacer $^{18,19}$. 

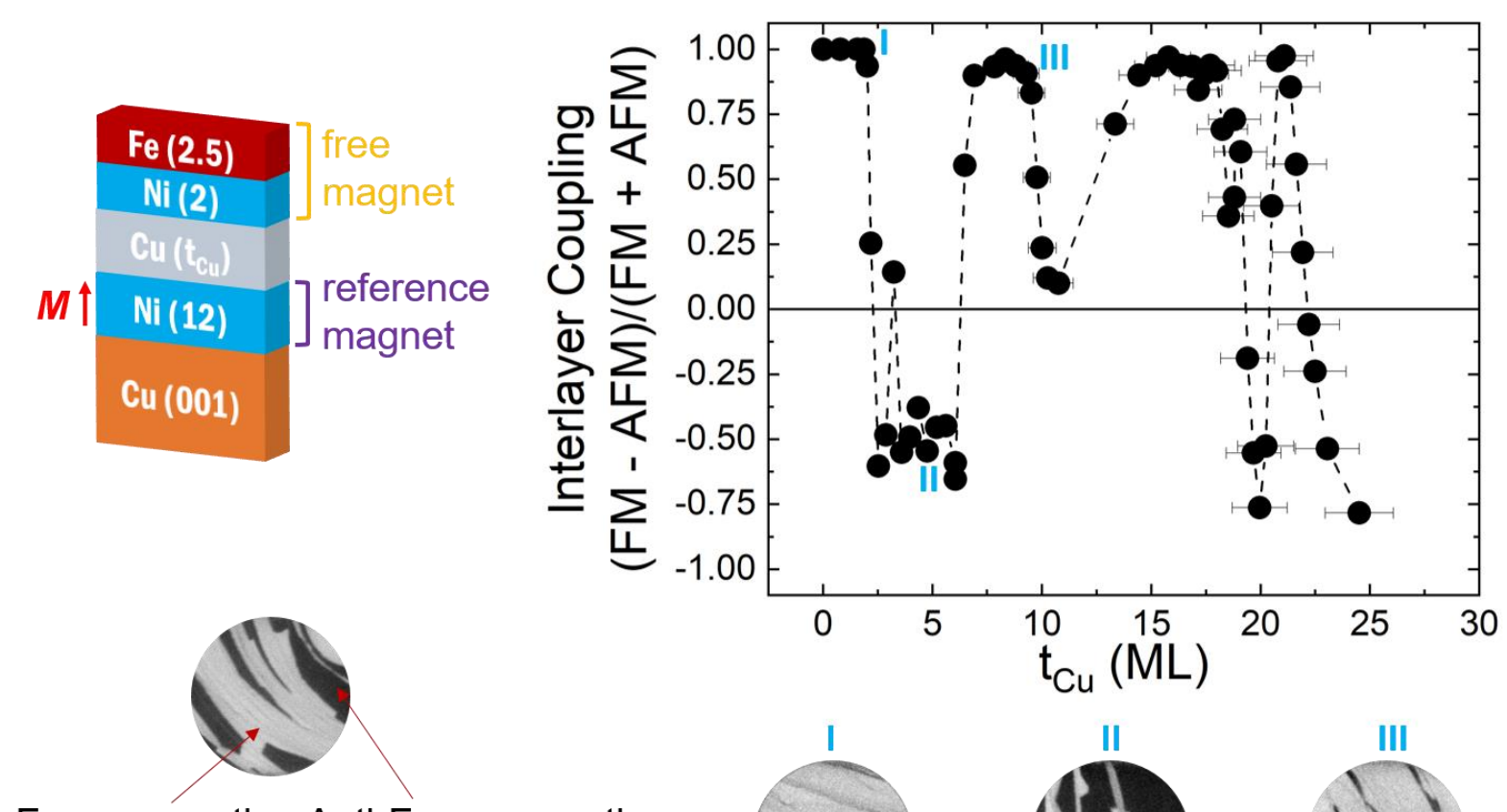

\section{Ferromagnetic Anti-Ferromagnetic alignment alignment} (FM) (AFM)

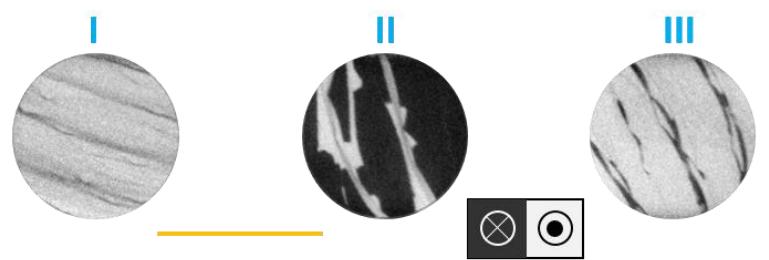

Figure S10. Interlayer exchange coupling in $\mathrm{Cu}(001) \backslash \mathrm{Ni}(12) \backslash \mathrm{Cu}\left(\mathbf{t}_{\mathrm{Cu}}\right) \backslash \mathrm{Ni}(2) \backslash \mathrm{Fe}(2.5)$. The qualitative behavior of the interlayer exchange coupling between the free and the reference magnet observed in a sample made of $\mathrm{Cu}(001) \backslash \mathrm{Ni}(12) \backslash \mathrm{Cu}\left(\mathrm{t}_{\mathrm{Cu}}\right) \backslash \mathrm{Ni}(2) \backslash \mathrm{Fe}(2.5)$ (all thicknesses in monolayer) is here reported. The yellow scale bar indicates $10 \mu \mathrm{m}$. 


\section{S9. Growth of wedged Cu spacer via shadow mask technique}

The sketch below quantifies the geometry of the wedge-growth setup.

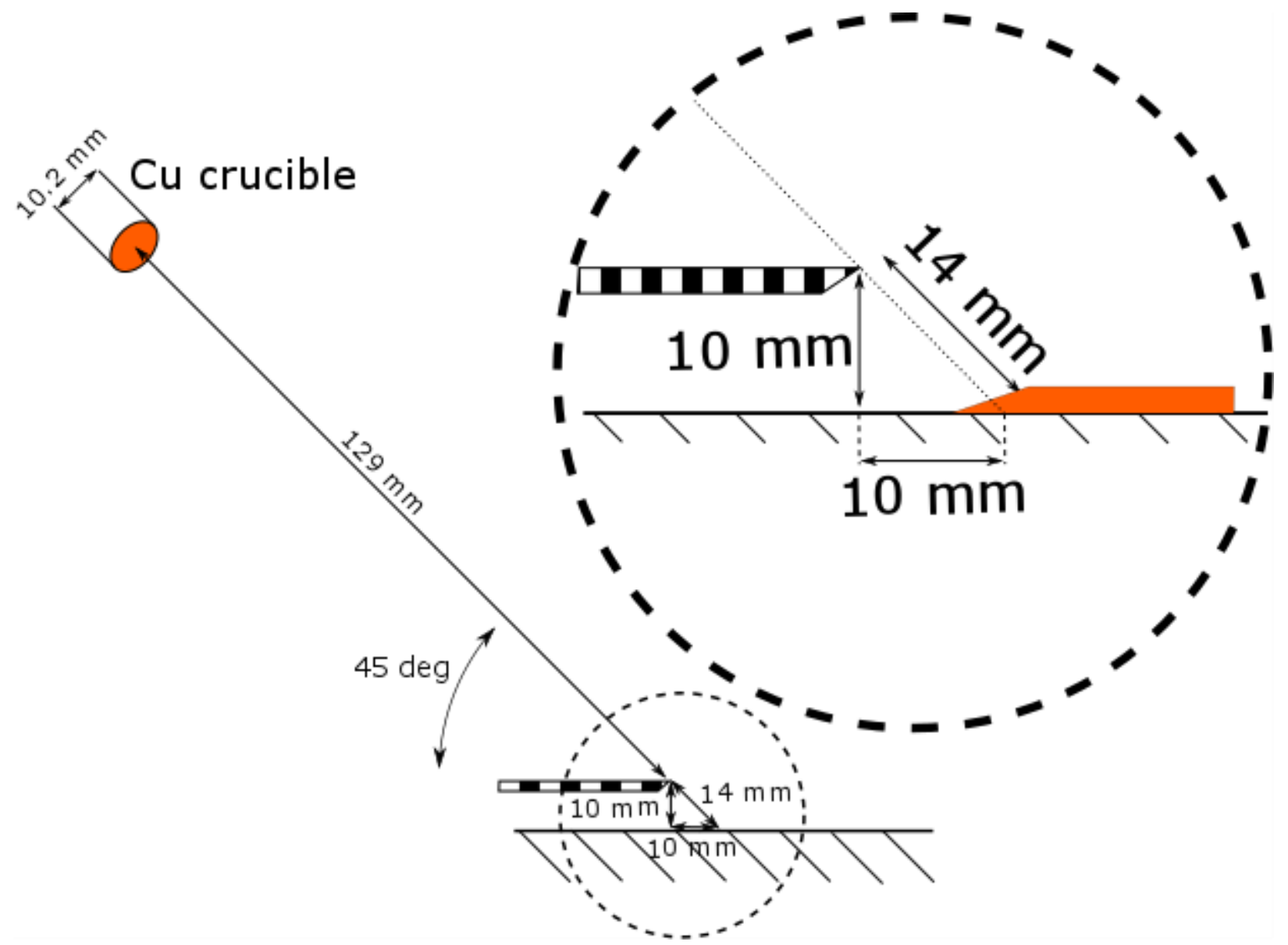

We can estimate the thickness profile of the wedge from the following model. If the Cu evaporator were a point-source with zero diameter, then the shadow of the knife-edge mask would be perfectly sharp, and the cross section shape of the "wedge" would be a step function separating zero thickness on the left versus full thickness on the right. In reality, the Cu evaporates off of the entire crucible surface, so the $\mathrm{Cu}$ source has the shape of a circular disk with diameter $10.2 \mathrm{~mm}$, which causes blurring of the knife-edge shadow. If the substrate surface were oriented orthogonal to the Cu vapor beam, then the width of the wedge would be $W=10.2 \times\left(\frac{10 \sqrt{2}}{129}\right)$. In reality the substrate surface is inclined $45^{\circ}$ with respect to the $\mathrm{Cu}$ flux direction, this stretches the wedge profile by a factor of $\sqrt{2}$, so that the width of the wedge is actually $W=10.2 \mathrm{~mm} \times \frac{10 \mathrm{~mm} \times 2}{129 \mathrm{~mm}}=1.58139 \mathrm{~mm}$.

As the Cu source has the shape of a circle, the function describing the shape of the thickness wedge is the integral over a circle: 


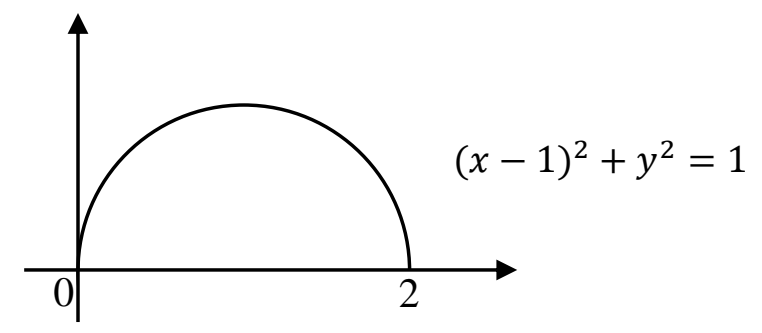

$$
\int y=\sqrt{2 x-x^{2}}
$$

We use the online integral calculator https://www.integral-calculator.com/ to integrate,

$$
A \int \sqrt{2 x-x^{2}}=A\left(\frac{x \sqrt{2 x-x^{2}}}{2}-\frac{\sqrt{2 x-x^{2}}}{2}-\frac{\arcsin \left(\frac{2-2 x}{2}\right)}{2}+C\right) \quad \text { Eq. [1] }
$$

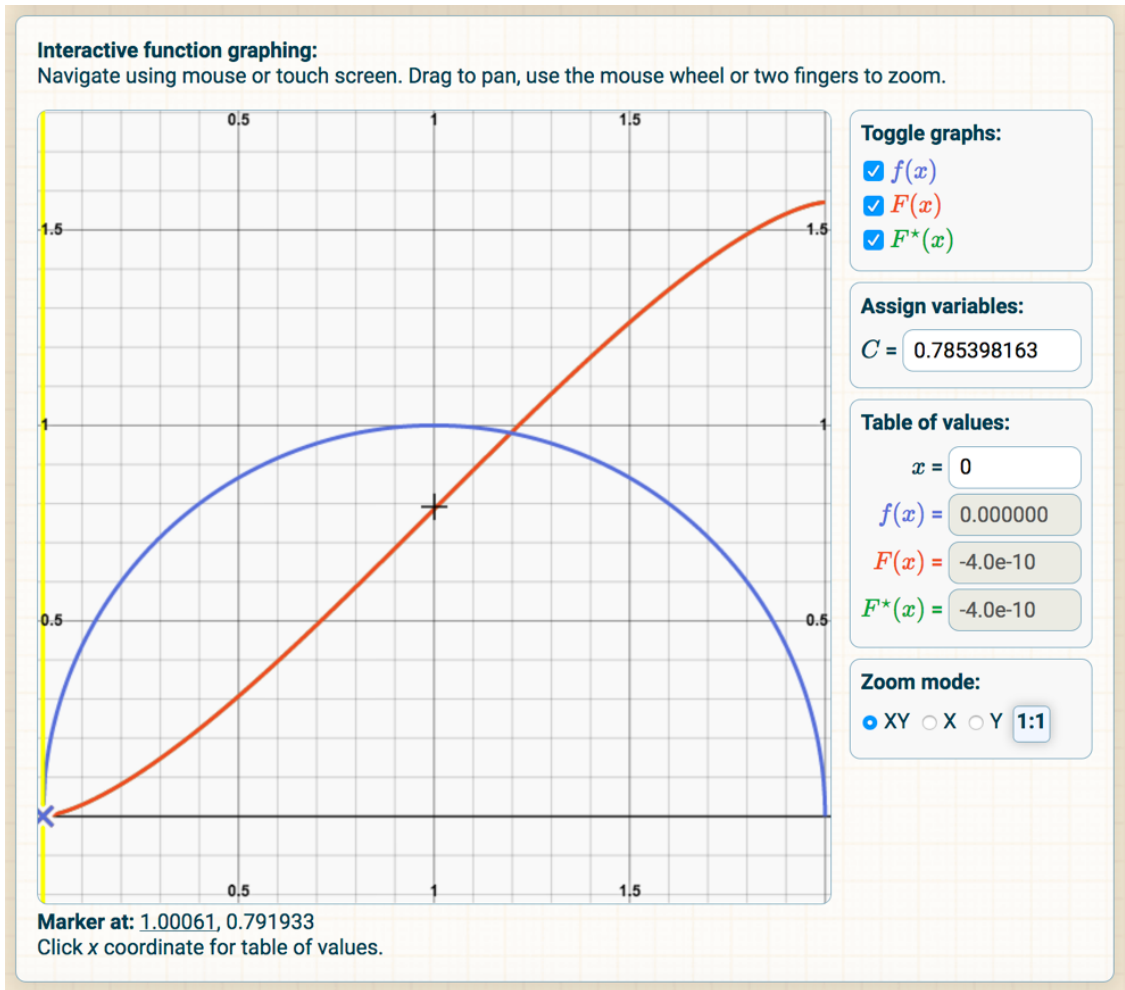

Equation [1] has free parameters $A$ and $C$ which take on the following values in our case. Thickness must be zero at the left edge of the wedge, i.e. at $x=0$. Therefore the $y$-offset parameter must be $C=$ 0.785398163 . For thickness to be 50 monolayers (equivalent to the total thickness deposited durng the depositin time) at the right edge of the wedge, the scaling factor must be $A=50 /(2 C)=31.830989$ 
In addition, to compute film thickness as a function of distance $x^{\prime}$ from the start-edge of the wedge in units of micrometer, we can scale the $x$-axis of Eq. [1] and use $x^{\prime}=x(1000 * 1.58 / 2)$.

Strictly speaking, the "thick" side of the wedge is approximately $1 \mathrm{~mm}$ further away from the source than the "thin" side. The resulting difference in deposition rate amounts to $(129+14.5)^{\wedge} 2 /(129+13.5)^{\wedge} 2=1.0141$, or a little over $1.4 \%$ error. To include this effect in the model, we can simply multiply the result based on equation [1] by the function

$f(x)=0.007-0.007 x$.

Result: The blue line in the graph below shows film thickness as a function of distance from the "thin" edge of the wedge, as predicted from this model, and a simple linear approximation is shown in orange.

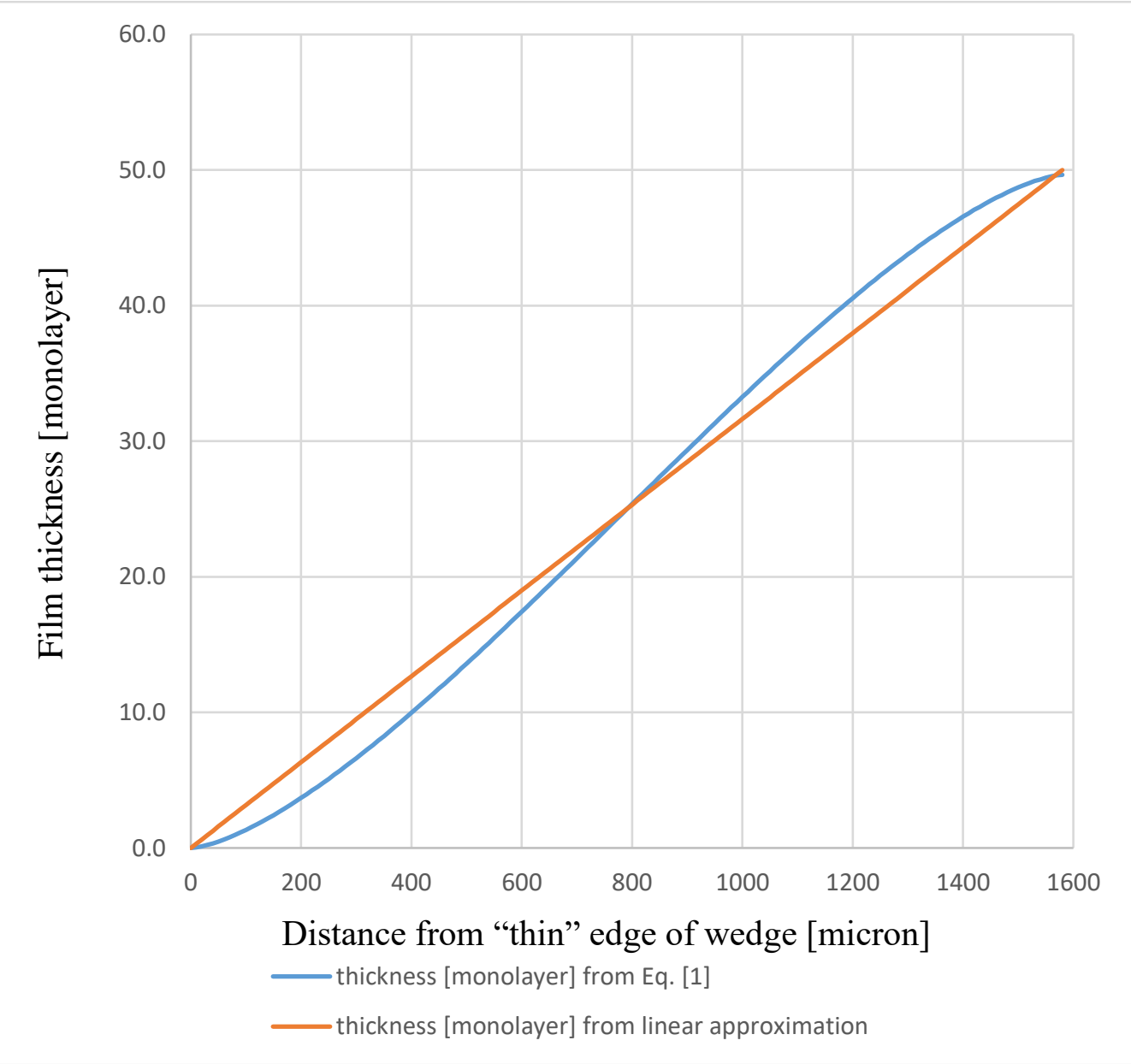


The table below shows the same data as plotted in the graph above, plus an additional column to quantify the error introduced by linear approximation. The linear approximation is problematic; it is particularly bad near the "thin" side of the wedge, where linear approximation introduces errors of as much as several hundred percent. For example, in the region around 100 micrometers away from the "thin" edge this linear approximation suggests film thickness of about 3 monolayers, while the more detailed model says that there's only about 1 monolayer of $\mathrm{Cu}$ in this area.

\section{position $\mathbf{x}^{\prime}$ [micrometer]}

\begin{tabular}{|c|c|c|c|}
\hline & $\begin{array}{l}\text { thickness [monolayer] } \\
\text { from Eq. [1] }\end{array}$ & $\begin{array}{l}\text { thickness [monolayer] } \\
\text { from linear } \\
\text { approximation }\end{array}$ & error of lin.approx. \\
\hline 0 & 0.0 & 0.0 & (N/A, div. zero) \\
\hline 10 & 0.0 & 0.3 & $638 \%$ \\
\hline 20 & 0.1 & 0.6 & $423 \%$ \\
\hline 30 & 0.2 & 0.9 & $328 \%$ \\
\hline 40 & 0.3 & 1.3 & $271 \%$ \\
\hline 50 & 0.5 & 1.6 & $233 \%$ \\
\hline 60 & 0.6 & 1.9 & $204 \%$ \\
\hline 70 & 0.8 & 2.2 & $182 \%$ \\
\hline 80 & 1.0 & 2.5 & $165 \%$ \\
\hline 90 & 1.1 & 2.8 & $150 \%$ \\
\hline 100 & 1.3 & 3.2 & $138 \%$ \\
\hline 110 & 1.5 & 3.5 & $127 \%$ \\
\hline 120 & 1.7 & 3.8 & $118 \%$ \\
\hline 130 & 2.0 & 4.1 & $110 \%$ \\
\hline 140 & 2.2 & 4.4 & $102 \%$ \\
\hline 150 & 2.4 & 4.7 & $96 \%$ \\
\hline 160 & 2.7 & 5.1 & $90 \%$ \\
\hline 170 & 2.9 & 5.4 & $85 \%$ \\
\hline 180 & 3.2 & 5.7 & $80 \%$ \\
\hline 190 & 3.4 & 6.0 & $76 \%$ \\
\hline 200 & 3.7 & 6.3 & $72 \%$ \\
\hline 210 & 4.0 & 6.6 & $68 \%$ \\
\hline 220 & 4.2 & 7.0 & $64 \%$ \\
\hline 230 & 4.5 & 7.3 & $61 \%$ \\
\hline 240 & 4.8 & 7.6 & $58 \%$ \\
\hline 250 & 5.1 & 7.9 & $55 \%$ \\
\hline 260 & 5.4 & 8.2 & $52 \%$ \\
\hline 270 & 5.7 & 8.5 & $50 \%$ \\
\hline 280 & 6.0 & 8.9 & $48 \%$ \\
\hline
\end{tabular}




\begin{tabular}{|c|c|c|c|}
\hline 290 & 6.3 & 9.2 & $45 \%$ \\
\hline 300 & 6.6 & 9.5 & $43 \%$ \\
\hline 310 & 6.9 & 9.8 & $41 \%$ \\
\hline 320 & 7.3 & 10.1 & $39 \%$ \\
\hline 330 & 7.6 & 10.4 & $37 \%$ \\
\hline 340 & 7.9 & 10.8 & $36 \%$ \\
\hline 350 & 8.3 & 11.1 & $34 \%$ \\
\hline 360 & 8.6 & 11.4 & $33 \%$ \\
\hline 370 & 8.9 & 11.7 & $31 \%$ \\
\hline 380 & 9.3 & 12.0 & $30 \%$ \\
\hline 390 & 9.6 & 12.3 & $28 \%$ \\
\hline 400 & 10.0 & 12.7 & $27 \%$ \\
\hline 410 & 10.3 & 13.0 & $26 \%$ \\
\hline 420 & 10.7 & 13.3 & $24 \%$ \\
\hline 430 & 11.0 & 13.6 & $23 \%$ \\
\hline 440 & 11.4 & 13.9 & $22 \%$ \\
\hline 450 & 11.8 & 14.2 & $21 \%$ \\
\hline 460 & 12.1 & 14.6 & $20 \%$ \\
\hline 470 & 12.5 & 14.9 & $19 \%$ \\
\hline 480 & 12.9 & 15.2 & $18 \%$ \\
\hline 490 & 13.2 & 15.5 & $17 \%$ \\
\hline 500 & 13.6 & 15.8 & $16 \%$ \\
\hline 510 & 14.0 & 16.1 & $15 \%$ \\
\hline 520 & 14.4 & 16.5 & $15 \%$ \\
\hline 530 & 14.7 & 16.8 & $14 \%$ \\
\hline 540 & 15.1 & 17.1 & $13 \%$ \\
\hline 550 & 15.5 & 17.4 & $12 \%$ \\
\hline 560 & 15.9 & 17.7 & $12 \%$ \\
\hline 570 & 16.3 & 18.0 & $11 \%$ \\
\hline 580 & 16.7 & 18.4 & $10 \%$ \\
\hline 590 & 17.0 & 18.7 & $10 \%$ \\
\hline 600 & 17.4 & 19.0 & $9 \%$ \\
\hline 610 & 17.8 & 19.3 & $8 \%$ \\
\hline 620 & 18.2 & 19.6 & $8 \%$ \\
\hline 630 & 18.6 & 19.9 & $7 \%$ \\
\hline 640 & 19.0 & 20.3 & $7 \%$ \\
\hline 650 & 19.4 & 20.6 & $6 \%$ \\
\hline 660 & 19.8 & 20.9 & $6 \%$ \\
\hline 670 & 20.2 & 21.2 & $5 \%$ \\
\hline 680 & 20.6 & 21.5 & $5 \%$ \\
\hline
\end{tabular}




\begin{tabular}{|c|c|c|c|}
\hline 690 & 21.0 & 21.8 & $4 \%$ \\
\hline 700 & 21.4 & 22.2 & $4 \%$ \\
\hline 710 & 21.8 & 22.5 & $3 \%$ \\
\hline 720 & 22.2 & 22.8 & $3 \%$ \\
\hline 730 & 22.6 & 23.1 & $2 \%$ \\
\hline 740 & 23.0 & 23.4 & $2 \%$ \\
\hline 750 & 23.4 & 23.7 & $2 \%$ \\
\hline 760 & 23.8 & 24.1 & $1 \%$ \\
\hline 770 & 24.2 & 24.4 & $1 \%$ \\
\hline 780 & 24.6 & 24.7 & $0 \%$ \\
\hline 790 & 25.0 & 25.0 & $0 \%$ \\
\hline 800 & 25.4 & 25.3 & $0 \%$ \\
\hline 810 & 25.8 & 25.6 & $-1 \%$ \\
\hline 820 & 26.2 & 25.9 & $-1 \%$ \\
\hline 830 & 26.6 & 26.3 & $-1 \%$ \\
\hline 840 & 27.0 & 26.6 & $-1 \%$ \\
\hline 850 & 27.4 & 26.9 & $-2 \%$ \\
\hline 860 & 27.8 & 27.2 & $-2 \%$ \\
\hline 870 & 28.2 & 27.5 & $-2 \%$ \\
\hline 880 & 28.6 & 27.8 & $-3 \%$ \\
\hline 890 & 29.0 & 28.2 & $-3 \%$ \\
\hline 900 & 29.4 & 28.5 & $-3 \%$ \\
\hline 910 & 29.8 & 28.8 & $-3 \%$ \\
\hline 920 & 30.1 & 29.1 & $-3 \%$ \\
\hline 930 & 30.5 & 29.4 & $-4 \%$ \\
\hline 940 & 30.9 & 29.7 & $-4 \%$ \\
\hline 950 & 31.3 & 30.1 & $-4 \%$ \\
\hline 960 & 31.7 & 30.4 & $-4 \%$ \\
\hline 970 & 32.1 & 30.7 & $-4 \%$ \\
\hline 980 & 32.5 & 31.0 & $-5 \%$ \\
\hline 990 & 32.9 & 31.3 & $-5 \%$ \\
\hline 1000 & 33.3 & 31.6 & $-5 \%$ \\
\hline 1010 & 33.6 & 32.0 & $-5 \%$ \\
\hline 1020 & 34.0 & 32.3 & $-5 \%$ \\
\hline 1030 & 34.4 & 32.6 & $-5 \%$ \\
\hline 1040 & 34.8 & 32.9 & $-5 \%$ \\
\hline 1050 & 35.2 & 33.2 & $-6 \%$ \\
\hline 1060 & 35.5 & 33.5 & $-6 \%$ \\
\hline 1070 & 35.9 & 33.9 & $-6 \%$ \\
\hline 1080 & 36.3 & 34.2 & $-6 \%$ \\
\hline
\end{tabular}




\begin{tabular}{|c|c|c|c|}
\hline 1090 & 36.7 & 34.5 & $-6 \%$ \\
\hline 1100 & 37.0 & 34.8 & $-6 \%$ \\
\hline 1110 & 37.4 & 35.1 & $-6 \%$ \\
\hline 1120 & 37.8 & 35.4 & $-6 \%$ \\
\hline 1130 & 38.1 & 35.8 & $-6 \%$ \\
\hline 1140 & 38.5 & 36.1 & $-6 \%$ \\
\hline 1150 & 38.8 & 36.4 & $-6 \%$ \\
\hline 1160 & 39.2 & 36.7 & $-6 \%$ \\
\hline 1170 & 39.5 & 37.0 & $-6 \%$ \\
\hline 1180 & 39.9 & 37.3 & $-6 \%$ \\
\hline 1190 & 40.2 & 37.7 & $-6 \%$ \\
\hline 1200 & 40.6 & 38.0 & $-6 \%$ \\
\hline 1210 & 40.9 & 38.3 & $-6 \%$ \\
\hline 1220 & 41.2 & 38.6 & $-6 \%$ \\
\hline 1230 & 41.6 & 38.9 & $-6 \%$ \\
\hline 1240 & 41.9 & 39.2 & $-6 \%$ \\
\hline 1250 & 42.2 & 39.6 & $-6 \%$ \\
\hline 1260 & 42.5 & 39.9 & $-6 \%$ \\
\hline 1270 & 42.9 & 40.2 & $-6 \%$ \\
\hline 1280 & 43.2 & 40.5 & $-6 \%$ \\
\hline 1290 & 43.5 & 40.8 & $-6 \%$ \\
\hline 1300 & 43.8 & 41.1 & $-6 \%$ \\
\hline 1310 & 44.1 & 41.5 & $-6 \%$ \\
\hline 1320 & 44.4 & 41.8 & $-6 \%$ \\
\hline 1330 & 44.7 & 42.1 & $-6 \%$ \\
\hline 1340 & 45.0 & 42.4 & $-6 \%$ \\
\hline 1350 & 45.2 & 42.7 & $-6 \%$ \\
\hline 1360 & 45.5 & 43.0 & $-5 \%$ \\
\hline 1370 & 45.8 & 43.4 & $-5 \%$ \\
\hline 1380 & 46.1 & 43.7 & $-5 \%$ \\
\hline 1390 & 46.3 & 44.0 & $-5 \%$ \\
\hline 1400 & 46.6 & 44.3 & $-5 \%$ \\
\hline 1410 & 46.8 & 44.6 & $-5 \%$ \\
\hline 1420 & 47.1 & 44.9 & $-5 \%$ \\
\hline 1430 & 47.3 & 45.3 & $-4 \%$ \\
\hline 1440 & 47.5 & 45.6 & $-4 \%$ \\
\hline 1450 & 47.7 & 45.9 & $-4 \%$ \\
\hline 1460 & 48.0 & 46.2 & $-4 \%$ \\
\hline 1470 & 48.2 & 46.5 & $-3 \%$ \\
\hline 1480 & 48.4 & 46.8 & $-3 \%$ \\
\hline
\end{tabular}




1490
1500
1510
1520
1530
1540
1550
1560
1570
1580

48.5

47.2

$-3 \%$

48.7

47.5

$-3 \%$

48.9

47.8

$-2 \%$

49.0

48.1

$-2 \%$

49.2

48.4

$-2 \%$

49.3

48.7

$-1 \%$

49.4

49.1

$-1 \%$

49.5

49.6

49.4

$0 \%$

49.6

49.7

$0 \%$

50.0

$1 \%$ 


\section{S10. Protocol for the extraction of the skyrmions area}

The protocol used for the extraction of the skyrmion size is the following:

1. Image the magnetic state of the top Fe layer;

2. Identify the stabilized domains which are larger than 8 pixels in area, so to distinguish real domains from spurious signals;

3. Extract the size of each identified skyrmion;

4. Use the area data extracted above for plotting its distribution (Fig. 3d in the main text);

5. Calculate the average area from the distributions above (Fig. 3e in the main text); 
S11. Estimation of the Curie temperatures of the reference and free magnetic layers and their effect on the IEC

The IEC is proportional to the product of the magnetization values in the two magnetic layers (IEC $\propto M_{1}$. $M_{2}$, where $M_{1}$ and $M_{2}$ are the magnetization in the reference and the free magnetic layers, respectively). It is important to keep in mind that DFT calculations report values at $0 \mathrm{~K}$, when the saturation magnetization of $\mathrm{Ni}(12)$ and $\mathrm{Ni}(2) \backslash \mathrm{Fe}(2)$ are at their maximum. However, in the experimental study the samples inside the microscope are held at a temperature of about $300 \mathrm{~K}$.

There is a lot of literature about the Curie temperatures, $T_{c}$, of magnetic thin films which can help us to better understand what the actual values of $M_{1}$ and $M_{2}$ are in our experimental case. Concerning the top $\mathrm{Ni}(2) \backslash \mathrm{Fe}(2)$ free layer, it is reported that a magnetic system made of $\mathrm{Cu}(001) \backslash \mathrm{Ni}(5.4) \backslash \mathrm{Fe}(2)$ has a $\mathrm{T}_{\mathrm{C}}$ of about $370 \mathrm{~K}^{20}$. While, concerning the bottom Ni(12) layer, F. Huang and colleagues ${ }^{21}$ reported a $\mathrm{T}_{\mathrm{C}}$ for $\mathrm{Cu}(001) \backslash \mathrm{Ni}(12.4)$ of $514 \mathrm{~K}$. Furthermore, is it well known that when a magnetic thin film is covered by a $\mathrm{Cu}$ layer (like for the bottom magnet in our samples) the $T_{C}$ of the film can be reduced significantly ${ }^{22}$. Indeed, it is reported that the $\mathrm{T}_{\mathrm{c}}$ of $\mathrm{Cu}(001) \backslash \mathrm{Fe}$ can be reduced by up to $150 \mathrm{~K}$ by the deposition of a $\mathrm{Cu}$ capping layer ${ }^{23}$. It is also reported that the $\mathrm{T}_{\mathrm{c}}$ of $\mathrm{Cu}(001) \backslash \mathrm{Ni}$ can be reduced up to $90 \mathrm{~K}$ by the presence of a Cu-cap, like in the case of $\mathrm{Cu}(001) \backslash \mathrm{Ni}(5)^{21}\left[\mathrm{~T}_{\mathrm{C}}=340 \mathrm{~K}\right]$ and $\mathrm{Cu}(001) \backslash \mathrm{Ni}(5) \backslash \mathrm{Cu}(25)^{24}\left[\mathrm{~T}_{\mathrm{C}}=250 \mathrm{~K}\right]$. Based on those results reported above, we estimate the Curie temperatures for the two magnetic layers in our samples to be: $\mathrm{T}_{\mathrm{C}}(\mathrm{Ni}(\mathbf{1 2}))=\mathbf{4 2 5} \mathrm{K} ; \mathrm{T}_{\mathrm{C}}([\mathrm{Ni}(\mathbf{2}) \backslash \mathrm{Fe}(2)])=370 \mathrm{~K}$.

Finally, we can estimate the magnetization value at room temperature $M_{i}(300 \mathrm{~K})$ via the following equation: $M(300)=M_{0} *\left(1-300 / T_{C}\right)^{\beta}$ (as reported by $F$. Huang et al. ${ }^{21}$ ), where $M_{0}$ is the magnetization value at $0 \mathrm{~K}$. Using the appropriate $\beta$ exponents for our thin films ${ }^{21}$ we extract: $\mathbf{M}_{\mathbf{1}}(\mathbf{3 0 0 K})=\mathbf{M}_{\mathbf{0}} *(\mathbf{1}-\mathbf{3 0 0} / \mathbf{4 2 5})^{\mathbf{0 . 4}}=$ $0.6 * M_{0} ; M_{2}(300 K)=M_{0} *(1-300 / 370)^{0.23}=0.68 * M_{0}$. This tells us that the actual magnetization values of the two magnets in our experiment are only a fraction of the values $0 \mathrm{~K}$ due to thermal excitations.

Accordingly, we expect the actual IEC to be much weaker than that predicted by DFT calculations. Indeed, using the estimated values of $M_{1}$ and $M_{2}$ at room temperature, then the actual coupling strength is only $\operatorname{IEC}(300 \mathrm{~K})=0.6 * 0.68 * \mathrm{IEC}_{0}=0.4 * \mathrm{IEC}_{0}$, where IEC $\mathrm{I}_{0}$ is the IEC at $0 \mathrm{~K}$. This estimation of the actual strength of the IEC in our samples, even if very approximate, is clearly suggesting that the effective magnetic fields extracted via DFT are an overestimate of the actual ones, which allow the stabilization of the observed magnetic skyrmions. 


\section{References}

S1. Kresse, G. \& Hafner, J. Ab initio molecular dynamics for liquid metals. Phys. Rev. B 1993, 47, 558561.

S2. Kresse, G. \& Hafner, J. Ab initio molecular-dynamics simulation of the liquid-metal amorphoussemiconductor transition in germanium. Phys. Rev. B 1994, 49, 14251-14269.

S3. Kresse, G. \& Furthmüller, J. Efficient iterative schemes for $a b$ initio total-energy calculations using a plane-wave basis set. Phys. Rev. B. 1996, 54, 11169-11186.

S4. Kresse, G. \& Furthmüller, J. Efficiency of ab-initio total energy calculations for metals and semiconductors using a plane-wave basis set. Comput. Mater. Sci. 1996, 6, 15-50.

S5. Kresse, G. \& Joubert, D. From ultrasoft pseudopotentials to the projector augmented-wave method. Phys. Rev. B 1999, 59, 1758-1775.

S6. Blöchl, P. E. Projector augmented-wave method. Phys. Rev. B 1994, 50, 17953-17979.

S7. Perdew, J. P.; Burke, K. \& Ernzerhof, M. Generalized gradient approximation made simple. Phys. Rev. Lett. 1996, 77, 3865-3868.

S8. FLEUR Project. Available at: http://www.flapw.de.

S9. Vosko, S. H.; Wilk, L. \& Nusair, M. Accurate spin-dependent electron liquid correlation energies for local spin density calculations: a critical analysis. Can. J. Phys. 1980, 58 (8), 1200-1211.

S10. Kurz, P.; Förster, F.; Nordström, L.; Bihlmayer, G. \& Blügel, S. Ab initio treatment of noncollinear magnets with the full-potential linearized augmented plane wave method. Phys. Rev. B 2004, 69, 024415.

S11. Heide, M., Bihlmayer, G. \& Blügel, S. Describing Dzyaloshinskii-Moriya spirals from first principles. Phys. B. Cond. Matt. 2009, 404, 2678.

S12. Bruno, P. Exchange Interaction Parameters and Adiabatic Spin-Wave Spectra of Ferromagnets: A "Renormalized Magnetic Force Theorem". Phys. Rev. Lett 2003, 90, 087205.

S13. Tian, Z.; Sander, D. \& Kirschner, J. Nonlinear magnetoelastic coupling of epitaxial layers of $\mathrm{Fe}, \mathrm{Co}$ and Ni on Ir(100). Phys. Rev. B 2009, 79, 024432.

S14. Altman, M. S.; Chung, W. F. \& Liu, C. H. LEEM Phase Contrast. Surf. Rev. Lett. 1998, 05, 11291141.

S15. Rougemaille, N. \& Schmid, A. K. Magnetic imaging with spin-polarized low-energy electron microscopy. Eur. Phys. J. Appl. Phys. 2010, 50, 20101.

S16. Chen, G. \& Schmid, A. K. Imaging and Tailoring the Chirality of Domain Walls in Magnetic Films. Adv. Mater. 2015, 27, 5738-5743.

S17. Davies, J. E.; Hellwig, O.; Fullerton, E. E.; Denbeaux, G.; Kortright, J. B. \& Liu, K. Magnetization reversal of Co/Pt multilayers: Microscopic origin of high-field magnetic irreversibility. Phys. Rev. B 2004, 70, 224434.

S18. Bruno, P. \& Chappert, C. 1993 Interlayer Exchange Coupling: RKKY Theory and Beyond. In: Farrow R.F.C., Dieny B., Donath M., Fert A., Hermsmeier B.D. (eds) Magnetism and Structure in Systems of Reduced Dimension. NATO ASI Series (Series B: Physics), vol 309. Springer, Boston, MA.

S19. Bruno, P. \& Chappert C. Oscillatory Coupling between Ferromagnetic Layers Separated by a Nonmagnetic Metal Spacer. Phys. Rev. Lett. 1991, 67, 1602.

S20. Won, C.; Wu, Y.Z.; Choi, J.; Kim, W.; Scholl, A.; Doran, A.; Owens, T.; Wu, J.; Jin, X.F.; Zhao, H.W., Qiu, Z.Q. Magnetic stripe melting at the spin reorientation transition in Fe/Ni/Cu(001). Phys. Rev. B 2005, 71, 224429.

S21. Huang, F.; Kief, M.T.; Mankey, G.J.; Willis, R.F. Magnetism in the few-monolayers limit: A surface magneto-optic Kerr-effect study of the magnetic behaviour of ultrathin films of $\mathrm{Co}, \mathrm{Ni}$, and $\mathrm{Co}-\mathrm{Ni}$ 
alloys on $\mathrm{Cu}(110)$ and $\mathrm{Cu}(111)$. Phys. Rev. B 1994, 49, 3962.

S22. Srivastava, P.; Wilhelm, F.; Ney, A.; Farle, M.; Wende, H.; Haack, H.; Ceballo, G.; Baberschke, K. Magnetic moments and Curie temperature of $\mathrm{Ni}$ and $\mathrm{Co}$ thin films and coupled trilayers. Phys. Rev. $B$ 1998, 58, 5701.

S23. Vollmer, R.; van Dijken, S.; Schleberger, M.; Kirschner, J. Dependence of the Curie temperature on the Cu cover layer in $\mathrm{x}-\mathrm{Cu} / \mathrm{Fe} / \mathrm{Cu}(001)$ sandwiches. Phys. Rev. B 2000, 61, 1303.

S24. Bovensiepen, U.; Wilhelm, F.; Srivastava, P.; Poulopoulos, P.; Farle, M.; Ney, A.; Baberschke, K. Two Susceptibility Maxima and Element Specific Magnetizations in Indirectly Coupled Ferromagnetic Layers. Phys. Rev. Lett. 1998, 81, 2368. 